\title{
Markov branching processes with disasters: extinction, survival and duality to $p$-jump processes
}

\author{
By Felix Hermann and Peter Pfaffelhuber \\ Albert-Ludwigs University Freiburg
}

\begin{abstract}
A $p$-jump process is a piecewise deterministic Markov process with jumps by a factor of $p$. We prove a limit theorem for such processes on the unit interval. Via duality with respect to probability generating functions, we deduce limiting results for the survival probabilities of time-homogeneous branching processes with arbitrary offspring distributions, underlying binomial disasters. Extending this method, we obtain corresponding results for time-inhomogeneous birth-death processes underlying time-dependent binomial disasters and continuous state branching processes with $p$-jumps.
\end{abstract}

\section{Introduction}

Consider a population evolving according to a branching process $\mathcal{Z}^{\prime}$. In addition to reproduction events, global events called disasters occur at some random times (independent of $\mathcal{Z}^{\prime}$ ) that kill off every individual alive with probability $1-p \in(0,1)$, independently of each other. The resulting process of population sizes $\mathcal{Z}$ will be called a branching process subject to binomial disasters with survival probability $p$. Provided no information regarding fitness of the individuals in terms of resistance against disasters, this binomial approach appears intuitive, since the survival events of single individuals are iid. Applications span from natural disasters as floods and droughts to effects of radiation treatment or chemotherapy on cancer cells as well as antibiotics on populations of bacteria. Also, Bernoulli sampling comes into mind as in the Lenski Experiment (cf. Casanova et al., 2016).

In the general setting of Bellman-Harris processes with non-lattice lifetime-distribution subject to binomial disasters, Kaplan et al. (1975) and Athreya and Kaplan (1976) have studied the almost sure asymptotic behaviour as well as asymptotics of the expectation of the population size and showed that such processes almost surely either go extinct or explode, giving necessary and sufficient conditions for extinction. They also computed the limit of the age-distribution on the set of explosion. In the special case of homogeneous birth-death processes with binomial disasters, Bühler and Puri (1989) obtained more explicit results regarding asymptotics and normalised limit distributions as well as the distribution of the extinction probability conditioned on the disaster times. Bartoszynski et al. (1989) added the analysis of extinction probabilities in a multi-type setting. Furthermore, Peng et al. (1993), Thilaka et al. (1998) and Kumar et al. (1998) studied single-type and multi-type population models underlying binomial disasters with survival probabilities depending on the time the last disaster occurred. These models reflect disasters like earthquakes where pressure builds up over time and increases severity. A more general disaster mechanism in a birth-death-scenario has been discussed by Brockwell et al. (1982), Brockwell

AMS 2000 subject classification. 60J80 (Primary) 60J75, 60F10 (Secondary).

Keywords and phrases. Piecewise deterministic Markov process, branching process, branching process with disasters, duality of Markov processes, extinction probability, survival probability 
(1985), Pakes (1986) and Pakes and Pollett (1989), where the absolute population decline after a catastrophe follows geometric, uniform or even an arbitrary distribution independent of the population size. Additionally, the rate of catastrophes is linear in the population size. For continuous state branching processes with disasters according to some intensity measure $\nu$, Bansaye et al. (2013) have studied the probability of extinction.

We will add to this literature of branching processes with disasters precise results for the asymptotic extinction probability at late times. As Theorem 2 shows, if extinction occurs, the survival probability decays exponentially at a rate which has a phase transition. We will also be dealing with the time-inhomogeneous case (see Theorem 3), and extinction probabilities for continuous state branching processes (CSBP) with binomial disasters (see Theorem 4).

The main technique we are going to use in our study is duality. Recall that duality of branching systems to a solution of a differential equation has particularly proven useful for continuous state branching processes and measure-valued processes. (See Etheridge, 2001 for an overview and the beginning of Section 4 for a brief introduction to this notion of duality.) Bringing this notion back to a branching process in continuous time (without disasters) $\mathcal{Z}$, where every individual branches at rate $\lambda$ and has offspring distribution with probability generating function (pgf) $h$, the distribution at time $t$ can be computed via the duality relation

$$
\mathbb{E}\left[x^{Z_{t}} \mid Z_{0}=z\right]=X_{t}^{z}
$$

where $X_{0}=x$ and $X_{t}$ solves

$$
\dot{X}=-\lambda(X-h(X))
$$

(cf. Athreva and Nev, 1972, Chapter III.3). We will generalize this equation in order to include binomial disasters. Here, the dual process will be a piecewise deterministic Markov process (PDMP) $\mathcal{X}$ on $[0,1]$, where $1-\mathcal{X}$ evolves according to (1.1) and jumps by a factor of $p$ at the rate of the disasters. Such processes will be called $p$-jump processes below. In Theorem 1 we will give general limit results for these processes, which become more precise and concise under concavity conditions shown in Corollary 2.4. These findings will then translate into limit and asymptotics results for survival and extinction probabilities of branching processes with binomial disasters in Theorem 2. The results of Theorem 2 will be expanded in two directions. First, we are dealing with the timeinhomogeneous case in Theorem 3, i.e. branching rate, pgf and disaster rate may depend on time. Here - using results of Kendall (1948) for the case without disasters - we are able to derive the pgf of a binary branching process subject to binomial disasters, conditioned on the times of disasters similar to the approach of Bühler and Puri (1989) for the homogeneous case. In this case, we also give in Proposition 4.11 some limits of pgfs. Second, we apply the duality technique to continuous state branching processes (CSBP) with binomial disasters. Here, we derive in Theorem 4 limit results for the extinction probabilities.

The manuscript is organised as follows. In Section 2, we give our main results on $p$-jump processes with Theorem 1 and Corollary 2.4. The results on (time-homogeneous) branching processes with disasters are collected in Theorem 2. The case of a birth-death process, i.e. a binary branching process, are given in Corollary 2.7 and extended further to the time-inhomogeneous case in Theorem 3. The CSBP with disasters is treated in Theorem 4 In Section 3, we will prove Theorem 1 and Corollary 2.4. The duality of branching processes with disasters and $p$-jump PDMP will be established in Section 4 , where we will also prove Theorem 2 For the time-inhomogeneous case, we first need in Section 4.2 some results on regularly varying functions, as collected in Theorem 4.6. which might be of interest in their own right. The proof of Theorem 3 is then given in Section 4.3 .

\section{Results}

\section{$2.1 \quad p$-jump Processes}

Let us begin by clarifying the notion of $p$-jump processes. 
Definition 2.1. Let $I=[0, v]$ for $v>0$ or $I=\mathbb{R}^{+}:=[0, \infty)$. Then, let $\alpha: I \rightarrow \mathbb{R}$ with $\alpha(0) \geq 0$ and $\alpha(v) \leq 0$, if $I=[0, v]$. Furthermore, let $p \in[0,1]$ and $\mathcal{X}$ be a right-continuous Markov process on $I$, that performs unit-rate jumps from a state $x$ to $p x$ and between jumps fulfils $\dot{X}_{t}=\alpha\left(X_{t}\right)$. Such a process has generator

$$
\mathcal{G}_{\mathcal{X}} f(x)=f(p x)-f(x)+\alpha(x) f^{\prime}(x)
$$

for $f \in \mathcal{C}^{1}(I)$ and is called a $p$-jump process with drift $\alpha$ on $I$.

\section{Remark 2.2.}

1. Note that such a process is uniquely characterised by $\alpha$, whenever $\dot{X}_{t}=\alpha\left(X_{t}\right)$ has a unique solution on $I \cap[\varepsilon, \infty)$ for every $\varepsilon>0$. To ensure this, we will use the Lipschitz-continuity conditions $\left(C_{1}\right)$ and $\left(C_{2}\right)$ in Theorem 1 . The bounds of $\alpha(0)$ and $\alpha(v)$ guarantee that the process does not leave the interval $I$, such that $\mathcal{X}$ is well-defined.

2. Due to its multiplicative jumps, such a process can only have 0 as an absorbing state. This happens, iff $\alpha(0)=0$.

3. 1-jump processes are deterministic, since their jumps have no effect, while 0-jump processes always jump to 0 . These two special cases will be left aside in Theorem 1 but considered in the following Corollary 2.4 for concave $\alpha$, where a more concise conclusion is possible.

At first, we present our most general limit results for $p$-jump processes.

Theorem 1 (Convergence of $p$-jump-processes). Let $I$ and $\alpha$ be as in Definition 2.1, $p \in(0,1)$ and $X_{0} \in I$. Also, suppose if $I=\mathbb{R}^{+}$that $s_{\alpha}:=\sup \{x: \alpha(x)>0\}<\infty$. Furthermore, let $\alpha_{0}^{\prime}:=\lim _{x \rightarrow 0} \frac{1}{x} \alpha(x)$ and assume that $\alpha$ satisfies one of the following:

$\left(C_{1}\right) \alpha$ is Lipschitz-continuous on I or

$\left(C_{2}\right) \alpha$ is Lipschitz-continuous on $I \cap\left[\varepsilon, \varepsilon^{-1}\right]$ for every $\varepsilon \in(0,1)$ and $\alpha_{0}^{\prime}=\infty$.

Then, there is a p-jump process $\mathcal{X}$ with drift $\alpha$ on I starting in $X_{0}$, such that, letting $\hat{\alpha}=$ $\sup _{x \in I \backslash\{0\}} \frac{1}{x} \alpha(x)$, the following statements hold:

1. If $\hat{\alpha}<\log \frac{1}{p}$, then $\mathbb{P}\left(X_{t} \stackrel{t \rightarrow \infty}{\longrightarrow} 0\right)=1$. Additionally, for the kth moment of $X_{t}, k \geq 1$, the following estimates hold:

$\left(U_{1}\right)$ In general (i.e. even for all $\hat{\alpha} \in \mathbb{R}$ ),

$$
\limsup _{t \rightarrow \infty} \frac{1}{t} \log \mathbb{E}\left[X_{t}^{k}\right] \leq-\left(1-p^{k}-\hat{\alpha} k\right) .
$$

$\left(U_{2}\right)$ If $\hat{\alpha}>p^{k} \log \frac{1}{p}$, letting $\lambda:=\frac{1}{\hat{\alpha}} \log \frac{1}{p}$, we obtain the stronger bound

$$
\limsup _{t \rightarrow \infty} \frac{1}{t} \log \mathbb{E}\left[X_{t}^{k}\right] \leq-\left(1-\frac{1}{\lambda}(1+\log \lambda)\right) .
$$

$\left(L_{1}\right)$ If there are $\delta \in \mathbb{R}$ and $\vartheta>0$ such that $\alpha(x) \geq \delta x-\vartheta x^{2}$ for all $x \in I$, such that $\delta \leq p^{k} \log \frac{1}{p}$, then

$$
\liminf _{t \rightarrow \infty} \frac{1}{t} \log \mathbb{E}\left[X_{t}^{k}\right] \geq-\left(1-p^{k}-\delta k\right)
$$

$\left(L_{2}\right)$ If $\delta$ in $\left(L_{1}\right)$ can be chosen positive, letting $\gamma:=\frac{1}{\delta} \log \frac{1}{p}$, we obtain

$$
\liminf _{t \rightarrow \infty} \frac{1}{t} \log \mathbb{E}\left[X_{t}^{k}\right] \geq-\left(1-\frac{1}{\gamma}(1+\log \gamma)\right),
$$

which is a stronger bound than $\left(L_{1}\right)$, if $\delta>p^{k} \log \frac{1}{p}$. 
2. If $\alpha_{0}^{\prime} \in\left(\log \frac{1}{p}, \infty\right]$, let $x_{\alpha}=\min \{x \in I \backslash\{0\}: \alpha(x)=0\}$. Then, $\mathcal{X}$ converges weakly and its limit $X_{\infty}$ satisfies $\mathbb{P}\left(X_{\infty} \in\left(0, x_{\alpha}\right]\right)=1, \mathbb{E}\left[X_{\infty}^{-1} \alpha\left(X_{\infty}\right)\right]=\log \frac{1}{p}$ and for every $k \geq 1$

$$
\mathbb{E}\left[X_{\infty}^{k}\right]=\frac{k}{1-p^{k}} \mathbb{E}\left[X_{\infty}^{k-1} \alpha\left(X_{\infty}\right)\right] .
$$

Also, the distribution of $X_{\infty}$ is the unique stationary distribution and for every bounded and measurable function $f: I \rightarrow \mathbb{R}$ almost surely

$$
\lim _{t \rightarrow \infty} \frac{1}{t} \int_{0}^{t} f\left(X_{s}\right) d s=\mathbb{E}\left[f\left(X_{\infty}\right)\right] .
$$

\section{Remark 2.3.}

1. Note that in Theorem $11 \alpha_{0}^{\prime}$ is finite, such that $\alpha(0)$ has to be 0 and it holds $\alpha_{0}^{\prime}=\alpha^{\prime}(0)$. Hence the similar notation. Case 2. accounts for both possibilities $\alpha(0)=0$ with $\alpha^{\prime}(0)=\infty$ as well as $\alpha(0)>0$.

2. Although the theorem shows existence of a $p$-jump process on $\mathbb{R}^{+}$, it is notable that such a process will only assume values in $\left[0, \max \left\{X_{0}, s_{\alpha}\right\}\right]$, since by definition it can never grow beyond $\sup \left(\left\{X_{0}\right\} \cup\{x: \alpha(x)>0\}\right)$.

In the case where $\alpha$ is concave, the bounds $\left(U_{i}\right)$ and $\left(L_{i}\right)$ align and the continuity conditions become evident such that we can give a much more concise result. Here, we will also include the cases $p=0$ and $p=1$.

Corollary 2.4. Let $p \in[0,1], I$ and $\alpha$ be as in Definition 2.1. Additionally assume that $\alpha$ is concave, $\alpha^{\prime \prime}(0) \in[-\infty, 0]$ exists and that either

$-I=[0, v]$ and $\alpha^{\prime}(v)>-\infty$ or

- $I=\mathbb{R}^{+}$and there is an $x>0$ such that $\alpha(x)=0$.

Then, letting $X_{0} \in I$ and $\alpha_{0}^{\prime}:=\lim _{x \rightarrow 0} \frac{1}{x} \alpha(x)$, there is a p-jump process $\mathcal{X}$ with drift $\alpha$ on $I$ starting in $X_{0}$ and satisfying:

1. If $\alpha_{0}^{\prime}<\log \frac{1}{p}$ or $p=0$, then $X_{t} \stackrel{t \rightarrow \infty}{\longrightarrow} 0$ almost surely. Also, for $k \geq 1$

$$
\lim _{t \rightarrow \infty}-\frac{1}{t} \log \mathbb{E}\left[X_{t}^{k}\right]= \begin{cases}1+\max \left\{0,-k \alpha_{0}^{\prime}\right\} & \text { if } p=0, \\ 1-p^{k}-k \alpha_{0}^{\prime} & \text { if } \alpha_{0}^{\prime} \leq p^{k} \log \frac{1}{p}, \\ 1-\frac{1}{\gamma}(1+\log \gamma) & \text { otherwise, }\end{cases}
$$

where $\gamma=\log \left(\frac{1}{p}\right) / \alpha_{0}^{\prime}$.

2. If $\alpha_{0}^{\prime}=\log \frac{1}{p}$, then $\frac{1}{t} \log \mathbb{E}\left[X_{t}^{k}\right] \stackrel{t \rightarrow \infty}{\longrightarrow} 0$ and $\lim \sup _{t} X_{t} \leq m_{\alpha}:=\sup \left\{x \in\left[0, x_{\alpha}\right]: \alpha(x)=\right.$ $\left.x \alpha_{0}^{\prime}\right\}$ almost surely. In particular, if $\alpha$ is strictly concave on an interval $(0, \varepsilon)$, then $X_{t} \rightarrow$ $m_{\alpha}=0$ almost surely.

3. If $\alpha_{0}^{\prime}>\log \frac{1}{p}$, then $x_{\alpha}:=\sup \{x: \alpha(x)>0\} \in(0, \infty), \mathcal{X}$ converges weakly and its limit $X_{\infty}$ satisfies $\mathbb{P}\left(X_{\infty} \in\left(0, x_{\alpha}\right]\right)=1, \mathbb{E}\left[X_{\infty}^{-1} \alpha\left(X_{\infty}\right)\right]=\log \frac{1}{p}$ and for every $k \geq 1$

$$
\mathbb{E}\left[X_{\infty}^{k}\right]=\frac{k}{1-p^{k}} \mathbb{E}\left[X_{\infty}^{k-1} \alpha\left(X_{\infty}\right)\right] .
$$

Also, the distribution of $X_{\infty}$ is the unique stationary distribution and for every bounded and measurable function $f: \mathbb{R}_{+} \rightarrow \mathbb{R}$ almost surely

$$
\lim _{t \rightarrow \infty} \frac{1}{t} \int_{0}^{t} f\left(X_{s}\right) d s=\mathbb{E}\left[f\left(X_{\infty}\right)\right] .
$$




\subsection{Branching Processes with Binomial Disasters}

Applying Corollary 2.4 to a $p$-jump process, via duality with respect to probability generating functions in Theorem 2 we obtain immediate limit results for the following class of branching processes with binomial disasters.

Definition 2.5. Let $\lambda>0, q=\left(q_{k}\right)_{k \geq 0}$ a distribution on $\mathbb{N}_{0}, \kappa>0$ and $p \in[0,1]$. A Markov process $\mathcal{Z}$ on $\mathbb{N}_{0}$ with generator

$$
\mathcal{G}_{\mathcal{Z}} f(z)=\lambda z \sum_{k \geq 0} q_{k}(f(z-1+k)-f(z))+\kappa \sum_{k=0}^{z}\left(\begin{array}{l}
z \\
k
\end{array}\right) p^{k}(1-p)^{z-k}(f(k)-f(z))
$$

for $f \in \mathcal{B}\left(\mathbb{N}_{0}\right)$, the set of real-valued, bounded functions on $\mathbb{N}$, is called a homogeneous branching process with death-rate $\lambda$ and offspring distribution $\left(q_{k}\right)$, subject to binomial disasters at rate $\kappa$ with survival probability $p$ and will be denoted by $\mathcal{Z}_{\lambda, q, \kappa, p}^{h}$.

Such a process describes the size of a population that behaves in the following way: Every individual dies with rate $\lambda$ and leaves behind a random number of offsprings distributed according to $\left(q_{k}\right)$. Independent of this growth mechanism, with rate $\kappa$ global events occur that kill off every individual alive at that time with probability $1-p$ independently of each other.

Theorem 2. Let $\lambda>0, q=\left(q_{k}\right)_{k \geq 0}$ a distribution on $\mathbb{N}_{0}$ with expectation $\mu:=\sum_{k} k q_{k}, \kappa>0$ and $p \in[0,1)$. Then, if $p=0, \mathcal{Z}$ goes extinct almost surely with

$$
\lim _{t \rightarrow \infty}-\frac{1}{t} \log \mathbb{P}\left(Z_{t}>0\right)=\kappa+\max \{\lambda(1-\mu), 0\} .
$$

Otherwise, letting $\nu=\lambda(\mu-1) /\left(\kappa \log \frac{1}{p}\right), \mathcal{Z}:=\mathcal{Z}_{\lambda, q, \kappa, p}^{h}$ satisfies

1. if $\nu \leq p, \mathcal{Z}$ goes extinct almost surely and

$$
\lim _{t \rightarrow \infty}-\frac{1}{t} \log \mathbb{P}\left(Z_{t}>0\right)=(1-p) \kappa-\lambda(\mu-1) .
$$

2. if $p<\nu \leq 1, \mathcal{Z}$ goes extinct almost surely and

$$
\lim _{t \rightarrow \infty}-\frac{1}{t} \log \mathbb{P}\left(Z_{t}>0\right)=\kappa\left(1-\nu-\nu \log \left(\frac{1}{\nu}\right)\right) .
$$

3. if $\nu>1$, then $\mathcal{Z}$ survives with positive probability, where

$$
0<\mathbb{P}\left(\lim _{t \rightarrow \infty} Z_{t}=\infty\right)=1-\mathbb{P}\left(\lim _{t \rightarrow \infty} Z_{t}=0\right)=\sum_{k=1}^{z}\left(\begin{array}{l}
z \\
k
\end{array}\right)(-1)^{k-1} \mathbb{E}\left[X^{k}\right]<1-x_{*}^{z_{0}},
$$

where $h(x)=\sum_{k} x^{k} q_{k}$ is the pgf of $q, x_{*}$ is the smallest fixed point of $h$ and $X$ is a random variable on $\left(0,1-x_{*}\right]$ satisfying $\mathbb{E}\left[X^{-1}(1-h(1-X))\right]=1+\frac{\kappa}{\lambda} \log \frac{1}{p}$ and

$$
\mathbb{E}\left[X^{k}\right]=\frac{\lambda k}{\lambda k+\kappa\left(1-p^{k}\right)} \mathbb{E}\left[X^{k-1}(1-h(1-X))\right] .
$$

\section{Remark 2.6.}

1. Rearranging the inequalities in terms of $\mu$, we obtain

$$
\text { 1. if } \mu \leq 1+\frac{\kappa p}{\lambda} \log \frac{1}{p} \text {, 2. if } 1+\frac{\kappa p}{\lambda} \log \frac{1}{p}<\mu \leq 1+\frac{\kappa}{\lambda} \log \frac{1}{p} \text {, } \quad \text { 3. if } \mu>1+\frac{\kappa}{\lambda} \log \frac{1}{p} \text {. }
$$

These give insight into how supercritical the underlying branching process has to be in order to survive the disasters.

Also, this formulation illustrates the continuity of the theorem in $p=1$ : Classical results for such processes without disasters (cf. Harris, 1963, Theorem 11.1, p.109) show that $\mathcal{Z}$ goes extinct almost surely if $\mu \leq 1$ with $-\frac{1}{t} \log \mathbb{P}\left(Z_{t}>0\right) \rightarrow \lambda(1-\mu)$ as $t \rightarrow \infty$, which aligns with 1., while for $\mu>1, \mathbb{P}\left(Z_{t} \rightarrow \infty\right)=1-\mathbb{P}\left(Z_{t} \rightarrow 0\right)=1-x_{*}^{z_{0}}$, which is the upper bound for the survival probability in (2.2) for $p<1$. 
2. While Kaplan et al. (1975) have already shown the almost sure extinction in 1. and 2. as well as the fact in 3. that $\mathcal{Z}$ almost surely either goes extinct or explodes, we offer an alternative proof via our duality results plus rates of convergence for the survival probability including the case $\mu=\infty$. Also, making use of (2.3), our result offers a way to compute the exact extinction probability in 3. Since the recursion in (2.3) depends on the offspring pgf $h$, in general this formula can be difficult to compute. Corollary 2.7, however, shows that in the example of homogeneous birth-death-processes it is feasible.

The following corollary applies Theorem 2 to birth-death-processes with disasters. This does not only provide a nice transition to the next theorem, but offers an example where (using the relation (2.3)) we can explicitly compute the survival probability.

Corollary 2.7. Let $\mathcal{Z}:=\left(Z_{t}\right)_{t}$ be a homogeneous birth-death-process with respective rates $b>0$ and $d \geq 0$ that underlies binomial disasters at a rate of $\kappa>0$ with survival probability $p \in(0,1)$.

1. If $b-d \leq \kappa p \log \frac{1}{p}, \mathcal{Z}$ goes extinct almost surely and

$$
\lim _{t \rightarrow \infty}-\frac{1}{t} \log \mathbb{P}\left(Z_{t}>0\right)=(1-p) \kappa-(b-d) .
$$

2. If $\kappa p \log \frac{1}{p}<b-d \leq \kappa \log \frac{1}{p}, \mathcal{Z}$ goes extinct almost surely and

$$
\lim _{t \rightarrow \infty}-\frac{1}{t} \log \mathbb{P}\left(Z_{t}>0\right)=\kappa-\frac{b-d}{\log \frac{1}{p}}\left(1+\log \left(\frac{\kappa \log \frac{1}{p}}{b-d}\right)\right) .
$$

3. If $b-d>\kappa \log \frac{1}{p}$, then $\mathbb{P}_{k}\left(\lim _{t \rightarrow \infty} Z_{t}=0\right)+\mathbb{P}_{k}\left(\lim _{t \rightarrow \infty} Z_{t}=\infty\right)=1$ and

$$
\mathbb{P}_{k}\left(\lim _{t \rightarrow \infty} Z_{t}=\infty\right)=\left(1-\frac{d+\kappa \log \frac{1}{p}}{b}\right) \sum_{\ell=1}^{k}\left(\begin{array}{l}
k \\
\ell
\end{array}\right)(-1)^{\ell-1} \prod_{m=1}^{\ell-1}\left(1-\frac{d m+\left(1-p^{m}\right) \kappa}{b m}\right) .
$$

Proof. First note that $\mathcal{Z}$ is a $\mathcal{Z}_{\lambda, q, \kappa, p}^{h}$-process with $\lambda=b+d, q_{0}=d /(b+d)$ and $q_{2}=b /(b+d)=$ $1-q_{0}$. Thus, $\lambda(\mu-1)=(b+d)\left(q_{2}-q_{0}\right)=b-d$ and $\nu=(b-d) /\left(\kappa \log \frac{1}{p}\right)$, already providing 1 . and 2. by insertion in Theorem 2. For 3. we derive $1-h(1-x)=1-q_{0}-q_{2}(1-x)^{2}=q_{2} x(2-x)$, yielding a simple recursion in (2.3) concluding the proof.

In Section 4.3 we will develop tools for the analysis of inhomogeneous birth-death processes with time-dependent disasters, generalising the setting of Corollary 2.7. For this, mind the following definition, where the birth, death and disaster rates $b, d, \kappa$ as well as the survival probability $p$ are now given as functions of $t$.

Definition 2.8. Let $b, d, \kappa: \mathbb{R}_{+} \rightarrow \mathbb{R}_{+}$and $p: \mathbb{R}_{+} \rightarrow[0,1]$, where we abbreviate $b_{s}:=b(s), d_{s}:=$ $d(s), \kappa_{s}:=\kappa(s)$ and $p_{s}:=p(s)$. A Markov process $\mathcal{Z}$ on $\mathbb{N}_{0}$ with time-dependent generator (see Section $4.7 \mathrm{~A}$ of Ethier and Kurta, 1986)

$$
\begin{array}{r}
\mathcal{G}_{\mathcal{Z}, t} f(z)=b_{t} z(f(z+1)-f(z))+d_{t} z(f(z-1)-f(z)) \\
+\kappa_{t} \sum_{k=0}^{z}\left(\begin{array}{l}
z \\
k
\end{array}\right) p_{t}^{k}\left(1-p_{t}\right)^{z-k}(f(k)-f(z))
\end{array}
$$

for $t \geq 0$ and $f \in \mathcal{B}\left(\mathbb{N}_{0}\right)$, is called an inhomogeneous birth-death-process with birth-rate $b$ and death-rate $d$, subject to binomial disasters with survival probability $p$ occurring at rate $\kappa$ and will be denoted by $\mathcal{Z}_{b, d, \kappa, p}^{i n}$.

Key to our approach is Lemma 4.9. which computes the conditioned pgf delivering some kind of stronger duality, enabling us with Proposition 4.11 to easily give pgf limit results in terms of that dual process. While these tools offer room for further generalisation (cf. Remark 4.12), we give the following theorem as an example of application, where we also make use of Theorem 4.6. 
Theorem 3. Let $b, d, \kappa$ be non-negative right-continuous functions on $\mathbb{R}_{+}$with left limits and $p: \mathbb{R}_{+} \rightarrow[0,1]$ left-continuous with right limits such that $p_{t}=0$ only if $\kappa_{t}=0$ and, letting $\Lambda_{\kappa}^{-1}(t):=\inf \left\{s>0: \int_{0}^{s} \kappa_{s} d s>t\right\}$, such that the map $-\log \left(p\left(\Lambda_{\kappa}^{-1}(\cdot)\right)\right)$ is regularly varying. Furthermore, let $h: \mathbb{R}_{+} \rightarrow \mathbb{R}_{+}$continuous and non-decreasing with $\lim _{t \rightarrow \infty} t^{-\alpha} h(t)=\infty$ for some $\alpha>0$, as well as $\iota \in\{-1,1\}$ such that

$$
\frac{1}{h(t)} \int_{0}^{t}\left(b_{s}-d_{s}-\kappa_{s} \log \left(\frac{1}{p_{s}}\right)\right) d s \stackrel{t \rightarrow \infty}{\longrightarrow} \iota .
$$

Then, $\mathcal{Z}:=\mathcal{Z}_{b, d, \kappa, p}^{i n}$ satisfies

1. if $\iota=1$ and for some $\varepsilon>0$ holds $\int_{0}^{\infty} e^{-(1-\varepsilon) h(s)} b_{s} d s<\infty$, then $\mathbb{P}\left(Z_{t} \stackrel{t \rightarrow \infty}{\longrightarrow} 0\right)<1$.

2. if $\iota=-1$ or for some $\varepsilon>0$ holds $\int_{0}^{\infty} e^{-(1+\varepsilon) h(s)} b_{s} d s=\infty$, then $\mathbb{P}\left(Z_{t} \stackrel{t \rightarrow \infty}{\longrightarrow} 0\right)=1$.

\section{Remark 2.9.}

1. For $h(t)=t$ and $b, d, \kappa, p$ constant, this result aligns with the homogeneous case (cf. Corollary 2.7).

2. The regular variation condition on $p$ and $\kappa$ is equivalent to the existence of $\beta \in \mathbb{R}$ and some slowly varying function $\ell$, such that $p(t)=\exp \left(-\Lambda_{\kappa}(t)^{\beta} \ell\left(\Lambda_{\kappa}(t)\right)\right.$. We need $t^{-\alpha} h(t) \rightarrow \infty$ for some $\alpha>0$ to handle the case $\beta=-1$ in which Theorem 4.6 is inconclusive. If $\beta \neq-1$, one may choose $\alpha=0$.

3. The condition that $\kappa=0$ whenever $p=0$ ensures that no terminal disasters occur, i.e. disasters that render $\mathcal{Z}$ extinct with probability 1 . Dropping this, $\int_{0}^{t} \kappa_{s} \log \left(1 / p_{s}\right) d s$ might no longer be finite. However, letting $\kappa_{t}^{-}:=\kappa_{t} \cdot \delta_{0, p_{t}}$ and $\kappa_{t}^{+}:=\kappa_{t}-\kappa_{t}^{-}$, it is possible to apply Theorem 3 to the process $\mathcal{Z}=\mathcal{Z}_{b, d, \kappa^{+}, p}$ without the terminal disasters and separately compute the probability $\pi_{\text {term }}$ that at least one terminal disaster occurs, which for a unit-rate Poisson process $\left(P_{t}\right)$ satisfies

$$
\pi_{\text {term }}=\mathbb{P}\left(P_{\int_{0}^{\infty} \kappa_{t}^{-} d t}>0\right)=1-\exp \left(-\int_{t: p(t)=0} \kappa_{t} d t\right) .
$$

Since the sets $\left\{\kappa^{+}>0\right\}$ and $\left\{\kappa^{-}>0\right\}$ are disjoint, the respective counts of disasters on these sets are independent. This implies that the terminal disasters only affect the positivity of the survival probability, if $\int_{t: p(t)=0} \kappa_{t} d t=\infty$.

4. The cases where a normalisation function $h$ as in Theorem 3 does not exist, are discussed in Remark 4.13 1. Rates of convergence for the survival probability in case 2. will briefly be discussed in Remark 4.132 .

\subsection{Continuous State Branching Processes with Binomial Disasters}

The application of $p$-jump processes is not limited to branching processes with discrete states. We will now discuss survival and extinction for continuous state branching processes (see e.g. Lambert, 2008 for an overview) with binomial disasters. A similar model is studied in Bansaye et al. (2013), where multiplicative jumps occur for any factor according to some intensity measure. Their Theorem 1 shows existence and uniqueness of the process we now define.

Definition 2.10. Let $N$ a measure on $\mathbb{R}_{+}$with $\int_{0}^{\infty} \min \left(y, y^{2}\right) N(d y)<\infty, b \in \mathbb{R}, c, \kappa \in \mathbb{R}_{+}$and $p \in(0,1)$. Then, the $\mathbb{R}_{+}$-valued Markov process with generator

$$
\mathcal{G}_{\mathcal{Z}} f(z)=b z f^{\prime}(z)+c z f^{\prime \prime}(z)+\kappa(f(p z)-f(z))+\int_{0}^{\infty}\left(f(z+y)-f(z)-y f^{\prime}(z)\right) z N(d y),
$$

for $f \in \mathcal{C}_{b}^{2}\left(\mathbb{R}_{+}\right)$(the space of bounded, twice continuously differentiable functions) is called the continuous state branching process with $p$-disasters and will be denoted by $\mathcal{Z}_{b, c, N, \kappa, p}^{c s}$. 
Viewing a continuous state branching process as a scaling limit of a discrete branching process, by the law of large numbers the equivalent of a binomial disaster would be a $p$-jump of the population size, represented by the term $\kappa(f(p z)-f(z))$ in $\mathcal{G}_{\mathcal{z}}$. The next Theorem is similar to (but less precise than) Corollary 6 of Bansaye et al. (2013), but does not need their restrictions $\int_{0}^{\infty} y^{2} N(d y)<\infty$ and $c>0$.

Theorem 4. Let $\mathcal{Z}=\mathcal{Z}_{b, c, N, \kappa, p}^{c s}$ be a continuous state branching process with $p$-disasters as in Definition 2.10 and let

$$
\alpha: \mathbb{R}_{+} \rightarrow \mathbb{R}, x \mapsto b x-c x^{2}-\int_{0}^{\infty}\left(e^{-x y}-1+x y\right) N(d y)
$$

satisfy $\lim \sup _{x \rightarrow \infty} x^{-(1+\varepsilon)} \alpha(x)<0$ for some $\varepsilon>0$.

1. If $b \leq \kappa \log \frac{1}{p}, Z_{t} \stackrel{t \rightarrow \infty}{\longrightarrow} 0$ almost surely and

$$
\lim _{t \rightarrow \infty}-\frac{1}{t} \log \mathbb{P}\left(Z_{t}>0\right)= \begin{cases}(1-p) \kappa-b & \text { if } b \leq \kappa p \log \frac{1}{p}, \\ \kappa-\frac{\kappa}{\gamma}(1+\log \gamma) & \text { otherwise, }\end{cases}
$$

where $\gamma:=\kappa \log \left(\frac{1}{p}\right) / b$.

2. If $b>\kappa \log \frac{1}{p}, \lim _{t \rightarrow \infty} \mathbb{P}\left(Z_{t}=0 \mid Z_{0}=z\right) \in\left(e^{-z \xi}, 1\right)$, where $\xi$ denotes the largest root of $\alpha$.

We will only give an outline of the proof, since its structure is illustrative for the proofs to come and it differs only in details.

Sketch of proof. Due to a rescaling argument, we just need to show the case of $\kappa=1$. Setting $H(x, z):=e^{-x z}$ and applying the generator to the function $z \mapsto H(x, z)$ for $x$ fixed gives

$$
\mathcal{G}_{\mathcal{Z}} H(x, .)(z)=\left(b x-c x^{2}-\int_{0}^{\infty}\left(e^{-x y}-1+x y\right) N(d y)\right) \frac{\partial H}{\partial x}(x, z)+H(x p, z)-H(x, z)
$$

In other words (cf. (D') at the beginning of Section 4 ), $\mathcal{Z}$ is dual to the $p$-jump process with drift $\alpha$. Now, note that $\xi=\bar{x}_{\alpha}<\infty$, since $\limsup _{x \rightarrow \infty} x^{-(1+\varepsilon)} \alpha(x)<0$ and thus, $\alpha(x) \rightarrow-\infty$ as $x \rightarrow \infty$. Hence, either $c>0$ or $N((0, \infty))>0$. In any case, for all $x>0$

$$
\alpha^{\prime \prime}(x)=-2 c-\int_{0}^{\infty} x^{2} e^{-x y} N(d y)<0
$$

and $\alpha$ is strictly concave. Hence, Corollary 2.4 applies with $\alpha_{0}^{\prime}=\alpha^{\prime}(0)=b$.

Since $\lim \sup _{x \rightarrow \infty} x^{-(1+\varepsilon)} \alpha(x)<0, \mathcal{X}$ comes down from infinity in the sense that $\mathcal{X}$ is well-defined in the limit of $X_{0} \rightarrow \infty$. It follows

$$
\mathbb{P}\left(Z_{t}=0 \mid Z_{0}=z\right)=\lim _{x \rightarrow \infty} \mathbb{E}\left[e^{-x Z_{t}} \mid Z_{0}=z\right]=\mathbb{E}\left[e^{-z X_{t}} \mid X_{0}=\infty\right] .
$$

Using that $x-\frac{x^{2}}{2} \leq 1-e^{-x} \leq x$ for all $x \geq 0$ to estimate the rates of convergence, Corollary 2.4 concludes the proof, where the almost sure convergence in 1 . comes from the fact that $\mathcal{Z}$ is a supermartingale. (This can be seen by computing the martingale given by $Z_{t} \exp \left(-\int_{0}^{t} \frac{\mathcal{G}_{\mathcal{Z}} \mathrm{id}\left(Z_{s}\right)}{Z_{s}} d s\right)$.)

Remark 2.11. In the case $b>\kappa \log \frac{1}{p}$, we have

$$
\mathbb{P}\left(Z_{t}=0 \mid Z_{0}=z\right) \stackrel{t \rightarrow \infty}{\longrightarrow} \mathbb{E}\left[e^{-z X_{\infty}}\right]=: L(z),
$$

the Laplace transform of the limit of $\mathcal{X}$. Using stationarity it follows from $\mathbb{E}\left[\mathcal{G}_{\mathcal{X}} f\left(X_{\infty}\right)\right]=0$ for $f(x)=e^{-z x}$, that $L$ has to satisfy the functional equation

$$
\kappa(L(p z)-L(z))-b z L^{\prime}(z)+c z L^{\prime \prime}(z)+z \int_{0}^{\infty} L(z+y)-L(z)+y L^{\prime}(z) N(d y)=0,
$$

which for appropriate $N$ might deliver a more precise result than Theorem 4 .2. 


\section{Piecewise Deterministic Markov Processes}

In this section, we start by proving Theorem 1. mainly by applying large deviation results for Poisson processes, given in Appendix \$, and the work of Bladt and Nielsen (2017) regarding regenerative processes. Using this, we prove Corollary 2.4 in Section 3.2 for concave $\alpha$ offering a more concise result including continuities for $p \in\{0,1\}$.

\subsection{Proof of Theorem 1}

Proof. At the very beginning, suppose that the theorem already holds for $p$-jump processes on $[0,1]$, let $\alpha$ be as in the assumptions, $s:=\max \left\{X_{0}, s_{\alpha}\right\}$ and consider $a:[0,1] \rightarrow \mathbb{R}^{+}, x \mapsto \alpha(s x) / s$. It is straightforward to show that $a$ satisfies the conditions of Definition 2.1 as well as the ones of Theorem 1 such that there is a $p$-jump process $\overline{\mathcal{X}}=\left(\bar{X}_{t}\right)$ with drift $a$ on $[0,1]$ starting in $s X_{0}$ for which the assertions of the theorem hold, where $a_{0}^{\prime}=\alpha_{0}^{\prime}, \hat{a}=\hat{\alpha}$ and $x_{a}=s x_{\alpha}$. Also, $a(x) \geq$ $\delta x-\vartheta x^{2}$ for all $x \in[0,1]$ iff $\alpha(x) \geq \delta x-\frac{\vartheta}{s} x^{2}$ for all $x \in[0, s]$. Considering that $t \mapsto X_{t}:=s_{\alpha} \bar{X}_{t}$ also performs $p$-multiplicative jumps at rate 1 and in betwteen satisfies $\dot{X}_{t}=s \cdot a\left(\bar{X}_{t}\right)=\alpha\left(X_{t}\right)$, we obtain that the theorem holds. Hence, without loss of generality, we assume for the rest of the proof that $I=[0,1]$.

Note that in $\left(C_{1}\right), \alpha$ is Lipschitz-continuous on the whole interval $[0,1]$, while in $\left(C_{2}\right)$, there is an $x^{+}>0$ such that $\alpha(x)>0$ for all $x \in\left(0, x^{+}\right]$. In the latter case, the initial value problem with $f^{\prime}=\alpha(f)$ and $f(0)>0$ is equivalent to the restriction $f^{\prime}=\left.\alpha\right|_{\left[\min \left\{f(0), x^{+}\right\}, 1\right]}(f)$, since $\alpha\left(\min \left\{f(0), x^{+}\right\}\right)>0$, where $\alpha$ is Lipschitz-continuous on $\left[\min \left\{f(0), x^{+}\right\}, 1\right]$. In either case, by the Picard-Lindelöf Theorem, for every deterministic piece on an interval between two jumps $\left[\tau_{k}, \tau_{k+1}\right)$ the initial value problem with $\dot{X}_{t}=\alpha\left(X_{t}\right)$ and $X_{\tau_{k}}:=p X_{\tau_{k}-}>0$ has a unique solution. Additionally, the bound $\alpha(1) \leq 0$ ensures that $\mathcal{X}$ does not leave the interval $[0,1]$. Thus, $\mathcal{X}$ is well-defined. (Also note that, since by definition $\alpha_{0}^{\prime} \leq \hat{\alpha},\left(C_{2}\right)$ only concerns case 2. Moreover, $\alpha_{0}^{\prime}<\infty$ only if $\alpha(0)=0$, in which case $\alpha_{0}^{\prime}=\alpha^{\prime}(0)$.)

1. Let $\beta(y):=e^{y} \alpha\left(e^{-y}\right)$ and $Y_{t}:=-\log X_{t} \in \mathbb{R}_{+}$. Then, the process $\mathcal{Y}:=\left(Y_{t}\right)_{t \geq 0}$ has the generator

$$
\begin{aligned}
\mathcal{G}_{\mathcal{Y}} g(y) & =\left(\mathcal{G}_{\mathcal{X}}(g \circ(-\log ))\right)\left(e^{-y}\right) \\
& =g\left(-\log \left(p e^{-y}\right)\right)-g(y)+\left.\alpha\left(e^{-y}\right) \cdot\left(-\frac{1}{x} g^{\prime}(-\log x)\right)\right|_{x=e^{-y}} \\
& =g\left(y+\log \frac{1}{p}\right)-g(y)-\beta(y) g^{\prime}(y) .
\end{aligned}
$$

The assertion implies that $\beta(y)=\frac{1}{e^{-y}} \alpha\left(e^{-y}\right) \leq \hat{\alpha}<\log \frac{1}{p}$ for all $y \in \mathbb{R}_{+}$. Letting $\left(P_{t}\right)$ be the unit-rate Poisson process jumping simultaneously with $\mathcal{Y}$, it follows that

$$
P_{t} \cdot \log \frac{1}{p}-t \hat{\alpha} \leq Y_{t}
$$

for all $t$, since the jumps are identical, the left side always starts in 0 and has point-wise inferior drift. The law of large numbers, giving us $\lim _{t \rightarrow \infty} P_{t} / t=1$ and thus almost surely $\lim _{i} \inf _{t}\left(Y_{t} / t\right) \geq$ $\log \frac{1}{p}-\hat{\alpha}>0$, shows that $-\log X_{t}=Y_{t} \longrightarrow_{t \rightarrow \infty} \infty$ with probability 1 .

$\left(U_{1}\right)$ Using the generator of $\mathcal{X}$ on $f(x)=x^{k}$, we obtain

$$
\frac{d}{d t} \mathbb{E}\left[X_{t}^{k}\right]=\mathbb{E}\left[\left(\mathcal{G}_{\mathcal{X}}(\cdot)^{k}\right)\left(X_{t}\right)\right]=\mathbb{E}\left[p^{k} X_{t}^{k}-X_{t}^{k}+\alpha\left(X_{t}\right) k X_{t}^{k-1}\right] \leq \mathbb{E}\left[X_{t}^{k}\right]\left(p^{k}-1+k \hat{\alpha}\right) .
$$

Considering Gronwall's inequality, it is straightforward to deduce $\left(U_{1}\right)$ (even for arbitrary $\hat{\alpha}$ ). 
$\left(U_{2}\right)$ For the (stronger) upper bound in the case of $\hat{\alpha}>p^{k} \log \frac{1}{p}$ we reuse (3.1) to compute

$$
\begin{aligned}
\mathbb{E}\left[X_{t}^{k}\right] & =\mathbb{E}\left[e^{-k Y_{t}}\right] \\
& \leq u(t):=\mathbb{E}\left[e^{-k\left(P_{t} \log (1 / p)-t \hat{\alpha}\right)} \wedge 1\right] \\
& =\mathbb{P}\left(P_{t} \leq \frac{\hat{\alpha}}{\log (1 / p)} t\right)+\sum_{\ell \geq \hat{\alpha} t /(\log (1 / p))} e^{k(\hat{\alpha} t-\log (1 / p) \ell)} e^{-t} \frac{t^{\ell}}{\ell !} \\
& =\underbrace{\mathbb{P}\left(P_{t} \leq \frac{t}{\lambda}\right)}_{=: u_{1}(t)}+\underbrace{e^{-t\left(1-p^{k}-\hat{\alpha} k\right)} \mathbb{P}\left(P_{p^{k} t} \geq \frac{1}{p^{k} \lambda} p^{k} t\right)}_{=: u_{2}(t)} .
\end{aligned}
$$

First, using A.2 of Lemma A.1 with $x=\frac{1}{\lambda}<1$, we compute

$$
-\frac{1}{t} \log u_{1}(t) \stackrel{t \rightarrow \infty}{\longrightarrow} 1-\frac{1}{\lambda}+\frac{1}{\lambda} \log \frac{1}{\lambda}=1-\frac{1}{\lambda}(1+\log \lambda)=: A_{\lambda} .
$$

For the second term, $u_{2}$, we see that $p^{k} \lambda<1$ and thus, by (A.1) of Lemma A.1.

$$
\begin{array}{r}
-\frac{1}{t} \log u_{2}(t) \stackrel{t \rightarrow \infty}{\longrightarrow} 1-p^{k}-\hat{\alpha} k+p^{k}\left(1-\frac{1}{p^{k} \lambda}+\frac{1}{p^{k} \lambda} \log \frac{1}{p^{k} \lambda}\right) \\
=1-\hat{\alpha} k-\frac{1}{\lambda}\left(1-\log \frac{1}{\lambda}-k \log \frac{1}{p}\right)=A_{\lambda} .
\end{array}
$$

Combining these two results, we obtain $\lim _{t \rightarrow \infty} \frac{1}{t} \log u(t)=-A_{\lambda}$, which equates to the desired bound.

To recognise that the bound in $\left(U_{2}\right)$ is in fact stronger than the one of $\left(U_{1}\right)$, we need to verify that $1-p^{k}-\hat{\alpha} k \leq A_{\lambda}$, if $p^{k} \lambda<1$. In this case, the function $h: x \mapsto x+\frac{1}{\lambda} \log \frac{1}{x}$ is strictly decreasing on $\left[p^{k}, \lambda^{-1}\right]$, since $h^{\prime}(x)=1-\frac{1}{\lambda x}$. Thus, inserting $\hat{\alpha}=\frac{1}{\lambda} \log \frac{1}{p}$, the difference satisfies

$$
\begin{aligned}
A_{\lambda}-\left(1-p^{k}-\hat{\alpha} k\right) & =p^{k}+\frac{1}{\lambda} \log \frac{1}{p^{k}}-\frac{1}{\lambda}(1+\log \lambda) \\
& =h\left(p^{k}\right)-h\left(\lambda^{-1}\right)>0 .
\end{aligned}
$$

$\left(L_{1}\right)$ and $\left(L_{2}\right)$ : Let $w:[0,1] \rightarrow \mathbb{R}, x \mapsto \delta x-\vartheta x^{2}$. Then, $\mathcal{X}$ is bounded below by a process $\mathcal{W}=\left(W_{t}\right)_{t}$ with generator

$$
\mathcal{G}_{\mathcal{W}} f(x)=f(p x)-f(x)+w(x) f^{\prime}(x),
$$

if $\mathcal{X}$ and $\mathcal{W}$ have equal initial values and are coupled in such a way that they jump simultaneously at the jump times of a Poisson process $\left(P_{t}\right)$. Now, we will show that the moments of $\mathcal{W}$ have the desired asymptotic properties, using that $\mathcal{W}$ can be represented explicitly via

$$
W_{t}=\frac{p^{P_{t}} e^{\delta t}}{X_{0}^{-1}+\vartheta \int_{0}^{t} p^{P_{s}} e^{\delta s} d s} .
$$

Surely, $\mathcal{W}$ starts in $X_{0}$ and has the desired jumps at the times of $P$, since $W_{t} / p^{P_{t}}$ is continuous. To verify the desired deterministic growth, let $\eta(t):=X_{0}^{-1}+\vartheta \int_{0}^{t} p^{P_{s}} e^{\delta s} d s$, the denominator of $W_{t}$, and assume that $P$ does not jump in an interval $(t-\varepsilon, t+\varepsilon)$. Then,

$$
\frac{d}{d t} W_{t}=\frac{p^{P_{t}} \delta e^{\delta t} \eta(t)-p^{P_{t}} e^{\delta t} \eta^{\prime}(t)}{\eta(t)^{2}}=W_{t} \cdot \frac{\delta \eta(t)-\vartheta p^{P_{t}} e^{\delta t}}{\eta(t)}=W_{t}\left(\delta-\vartheta W_{t}\right)=w\left(W_{t}\right) .
$$


Next, we consider the process $\overline{\mathcal{W}}$ that arises from $\mathcal{W}$ by exchanging for every $t$ the path $\left(P_{s}\right)_{0 \leq s \leq t}$ with its time-reversal $\left(\bar{P}_{s}^{t}\right)_{0 \leq s \leq t}$ via $\bar{P}_{s}^{t}:=P_{t}-P_{t-s}$, given by

$$
\begin{aligned}
\bar{W}_{t} & =\frac{p^{\bar{P}_{t}^{t}} e^{\delta t}}{X_{0}^{-1}+\vartheta \int_{0}^{t} p^{\bar{P}_{s}^{t}} e^{\delta s} d s}=\frac{1}{X_{0}^{-1} p^{-P_{t}} e^{-\delta t}+\vartheta \int_{0}^{t} p^{-P_{t-s}} e^{-\delta(t-s)} d s} \\
& =\left(X_{0}^{-1} p^{-P_{t}} e^{-\delta t}+\vartheta \int_{0}^{t} p^{-P_{s}} e^{-\delta s} d s\right)^{-1} .
\end{aligned}
$$

Now, since $\left(P_{s}\right)_{0 \leq s \leq t}$ is equally distributed as $\left(\bar{P}_{s+}\right)_{0 \leq s \leq t}$ for every $t>0$, where $\bar{P}_{s+}:=\lim _{r \downarrow s} \bar{P}_{r}$ denotes the right-side limit, we also have that $W_{t} \stackrel{d}{=} \bar{W}_{t}$ for all $t$ and in particular $\mathbb{E}\left[W_{t}^{k}\right]=\mathbb{E}\left[\bar{W}_{t}^{k}\right]$. Also note that, in contrast to $W_{t}, \bar{W}_{t}$ decreases if the path of $\left(P_{t}\right)$ increases pointwise. Now, using this and A.3) of Lemma A.1, we obtain for $\left(L_{2}\right)$ (even for arbitrary $\delta>0$ ), considering that $\delta \leq \hat{\alpha}$ and thus $\gamma \geq \frac{1}{\hat{\alpha}} \log \frac{1}{p}>1$ as well as $\delta=\frac{1}{\gamma} \log \frac{1}{p}$,

$$
\begin{aligned}
\frac{1}{t} \log \mathbb{E}\left[X_{t}^{k}\right] & \geq \frac{1}{t} \log \mathbb{E}\left[\bar{W}_{t}^{k}\right] \\
& =\frac{1}{t} \log \mathbb{E}\left[\left(X_{0}^{-1} p^{-P_{t}} e^{-\delta t}+\vartheta \int_{0}^{t} p^{-P_{s}} e^{-\delta s} d s\right)^{-k}\right] \\
& \geq \frac{1}{t} \log \mathbb{E}\left[\left(X_{0}^{-1} p^{-P_{t}} e^{-\delta t}+\vartheta \int_{0}^{t} p^{-P_{s}} e^{-\delta s} d s\right)^{-k}, P_{s} \leq \frac{s}{\gamma} \text { for all } s \leq t\right] \\
& \geq \frac{1}{t} \log \left(\left(X_{0}^{-1} e^{\left(\frac{1}{\gamma} \log \frac{1}{p}-\delta\right) t}+\vartheta \int_{0}^{t} e^{\left(\frac{1}{\gamma} \log \frac{1}{p}-\delta\right) s} d s\right)^{-k} \mathbb{P}\left(P_{s} \leq \frac{s}{\gamma} \text { for all } s \leq t\right)\right) \\
& \stackrel{t \rightarrow \infty}{\longrightarrow}-k \cdot 0-\left(1-\frac{1}{\gamma}(1+\log \gamma)\right)=A_{\gamma} .
\end{aligned}
$$

For the case $\delta \leq p^{k} \log \frac{1}{p}$, we deduce analogously

$$
\begin{aligned}
\frac{1}{t} \log \mathbb{E}\left[X_{t}^{k}\right] & \geq \frac{1}{t} \log \mathbb{E}\left[\left(X_{0}^{-1} p^{-P_{t}} e^{-\delta t}+\vartheta \int_{0}^{t} p^{-P_{s}} e^{-\delta s} d s\right)^{-k}\right] \\
& \geq \frac{1}{t} \log \left(\left(X_{0}^{-1} e^{\left(p^{k} \log \frac{1}{p}-\delta\right) t}+\vartheta \int_{0}^{t} e^{\left(p^{k} \log \frac{1}{p}-\delta\right) s} d s\right)^{-k} \mathbb{P}\left(P_{s} \leq p^{k} s \text { for all } s \leq t\right)\right) \\
& \stackrel{t \rightarrow \infty}{\longrightarrow}-k\left(p^{k} \log \frac{1}{p}-\delta\right)-\left(1-p^{k}+p^{k} \log \left(p^{k}\right)\right)=\delta k-\left(1-p^{k}\right) .
\end{aligned}
$$

With the same argument as at the end of the proof of $\left(U_{2}\right)$, one can recognise that the bound in $\left(L_{1}\right)$ is stronger than $A_{\gamma}$ if $\delta \leq p^{k} \log \frac{1}{p}$.

2. Recalling that $Y_{t}:=-\log X_{t}$ and letting $T_{z}:=\inf \left\{t>0: Y_{t}=z\right\}$, we show that (i) there is $z \geq 0$ such that $\mathbb{E}\left[T_{z} \mid Y_{0}=z\right]<\infty$ and (ii) $\mathbb{P}\left(T_{z}<\infty \mid Y_{0}=y\right)=1$ for all $y \geq 0$ :

Since $\alpha_{0}^{\prime}>\log \frac{1}{p}$, there have to be $\xi>0$ and $\varepsilon>0$ such that $\alpha(x) / x \geq(1+\varepsilon) \log \frac{1}{p}$ for all $x \in(0, \xi]$. Setting $z=-\log \xi<\infty$, we see that $\beta(y) \geq(1+\varepsilon) \log \frac{1}{p}=: \zeta$ for all $y \geq z$. We define

$$
S:=S_{(z, z+\log (1 / p)]}:=\inf \left\{t: z<Y_{t} \leq z+\log (1 / p)\right\} .
$$

Then, $\mathbb{E}\left[S \mid Y_{0}=y\right]<\infty$ for all $y \leq z$. Indeed, the probability for at least $z / \log (1 / p)$ jumps in some small time interval of length $\varepsilon^{\prime}>0$ is positive. After the first such time interval we can be sure that $S$ has occurred. By finiteness of first moments of geometric distributions, $\mathbb{E}\left[S \mid Y_{0}=y\right]<\infty$ follows. By a restart argument, we have to show that $\mathbb{E}\left[T_{z} \mid Y_{0}=y\right]<\infty$ for all $z<y \leq z+\log (1 / p)$, which will be done by using a comparison argument. For this, let $\mathcal{R}=\left(R_{t}\right)_{t \geq 0}$ be a process with generator

$$
\mathcal{G}_{\mathcal{R}} g(y)=g(y+\log (1 / p))-g(y)-\zeta g^{\prime}(y) .
$$


If $z<R_{0}=Y_{0} \leq z+\log (1 / p)$, then - using the same Poisson processes for $\mathcal{Y}$ and $\mathcal{R}$ - we have that $T_{z} \leq T_{z}^{\mathcal{R}}:=\inf \left\{t \geq 0: R_{t}=z\right\}$ since $\beta(y) \geq \zeta$ for $y \geq z$. Analogously to the argument following (3.1), we see that $R_{t} \rightarrow-\infty$ as $t \rightarrow \infty$ almost surely, which implies that $T_{z}^{\mathcal{R}}<\infty$. Since $\left(R_{t}-R_{0}+t(\zeta-\log (1 / p))\right)_{t \geq 0}$ is a martingale, we have by optional stopping that $\mathbb{E}\left[R_{0}-R_{T_{z}^{\mathcal{R}}}\right]=R_{0}-z=(\zeta-\log (1 / p)) \mathbb{E}\left[T_{z}^{\mathcal{R}}\right]=\varepsilon \log (1 / p) \mathbb{E}\left[T_{z}^{\mathcal{R}}\right]$, hence $\mathbb{E}\left[T_{z}^{\mathcal{R}} \mid Y_{0}=R_{0}\right] \leq 1 / \varepsilon<\infty$. It is now straightforward to obtain the properties (i) and (ii).

Now, by (i) and homogeneity, $\mathcal{Y}$ is a positively recurrent delayed regenerative process in the sense of Definition 7.1.1 in (Bladt and Nielsen, 2017, p. 387) with regeneration cycles starting at the state $z$ (and so is $\mathcal{X}$ with cycles starting in $\xi$ ). By (ii), the delay is almost surely finite. Hence, Theorem 7.1.4 in (Bladt and Nielsen, 2017, p. 388) gives us weak convergence of $\mathcal{Y}$ to a finite random variable $Y_{\infty}$ and thus, also $\mathcal{X}$ has a weak limit $X_{\infty}:=e^{-Y_{\infty}}>0$. Furthermore, recalling that $x_{\alpha}=\min \{x \in(0,1]: \alpha(x)=0\}$, this is well-defined since $\alpha$ is positive on $(0, \xi)$ and Lipschitzcontinuous on $[\xi, 1], \alpha_{0}^{\prime}>0$ and $\alpha(1) \leq 0$. This implies that the hitting time of $\left[0, x_{\alpha}\right)$ of $\mathcal{X}$ is almost surely finite. After that, $\mathcal{X}$ will never hit $\left[x_{\alpha}, 1\right]$ again, since it will always jump before it can reach $x_{\alpha}$. Thus, $\mathbb{P}\left(X_{\infty} \in\left(0, x_{\alpha}\right)\right)=1$.

If $f:[0,1] \rightarrow \mathbb{R}$ is measurable and bounded, Theorem 7.1.6 of (Bladt and Nielsen, 2017, p. 391) as well as the weak convergence give us that almost surely

$$
\lim _{t \rightarrow \infty} \frac{1}{t} \int_{0}^{t} f\left(X_{s}\right) d s=\lim _{t \rightarrow \infty} \frac{1}{t} \int_{0}^{t} \mathbb{E}\left[f\left(X_{s}\right)\right] d s=\mathbb{E}\left[f\left(X_{\infty}\right)\right] .
$$

Since for $t>0$ and a continuous and bounded function $f$ the distribution $\mu$ of $X_{\infty}$ satisfies

$$
\mathbb{E}_{\mu}\left[f\left(X_{t}\right)\right]=\mathbb{E}\left[\mathbb{E}_{X_{\infty}}\left[f\left(X_{t}\right)\right]\right]=\lim _{s \rightarrow \infty} \mathbb{E}\left[\mathbb{E}_{X_{s}}\left[f\left(X_{t}\right)\right]\right]=\lim _{s \rightarrow \infty} \mathbb{E}\left[f\left(X_{t+s}\right)\right]=\mathbb{E}\left[f\left(X_{\infty}\right)\right],
$$

$\mu$ is a stationary distribution. Letting $\nu$ be a stationary distribution of $\mathcal{X}$ we obtain from (3.5) that for all $t$

$$
\mathbb{E}_{\nu}\left[f\left(X_{1}\right)\right]=\frac{1}{t} \int_{0}^{t} \mathbb{E}_{\nu}\left[f\left(X_{s}\right)\right] d s=\mathbb{E}\left[f\left(X_{\infty}\right)\right],
$$

by which the stationary distribution must be unique. In particular, by stationarity $\mathbb{E}\left[\mathcal{G}_{\mathcal{X}} f\left(X_{\infty}\right)\right]=$ 0 holds. Hence, choosing $f(x)=\log (\rho+x)$ for $\rho>0$, we obtain

$$
0=\mathbb{E}\left[\log \left(\frac{\rho+p X_{\infty}}{\rho+X_{\infty}}\right)+\frac{\alpha\left(X_{\infty}\right)}{\rho+X_{\infty}}\right] \stackrel{\rho \rightarrow 0}{\longrightarrow}-\log \left(\frac{1}{p}\right)+\mathbb{E}\left[X_{\infty}^{-1} \alpha\left(X_{\infty}\right)\right]
$$

by monotone convergence, since $X_{\infty}>0$ almost surely. (Note here, that this argument works for both cases $\left(C_{1}\right)$ and $\left(C_{2}\right)$.) On the other hand, choosing $f(x)=x^{k}$, it follows

$$
0=\mathbb{E}\left[\left(p X_{\infty}\right)^{k}-X_{\infty}^{k}+\alpha\left(X_{\infty}\right) k X_{\infty}^{k-1}\right],
$$

which implies $\mathbb{E}\left[X_{\infty}^{k}\right]=\frac{k}{1-p^{k}} \mathbb{E}\left[X_{\infty}^{k-1} \alpha\left(X_{\infty}\right)\right]$.

\section{Remark 3.1.}

1. For $\alpha_{0}^{\prime}<\log \frac{1}{p}<\hat{\alpha}$, Theorem 1 is inconclusive. The weak estimates using $\hat{\alpha}$ in (3.1) and (3.2) offer room for improvement. Also note here that for the application of $\left(L_{1}\right)$ and $\left(L_{2}\right)$ for $\alpha(0)=0$, one can often choose $\delta=\alpha^{\prime}(0)=\alpha_{0}^{\prime}$.

2. Using the notion of strong large deviations, e.g. Chaganty and Sethuraman, 1993, Theorem 3.5, p.1868), one can compute exact asymptotics:

(a) If $x>1$, then $\mathbb{P}\left(P_{t} \geq x t\right) \sim \frac{\sqrt{x}}{x-1} \cdot \frac{e^{-t(1-x+x \log x)}}{\sqrt{2 \pi t}}$,
(b) If $0<x<1$, then $\mathbb{P}\left(P_{t} \leq x t\right) \sim \frac{1}{(1-x) \sqrt{x}} \cdot \frac{e^{-t(1-x+x \log x)}}{\sqrt{2 \pi t}}$, 
where $\sim$ denotes asymptotic equivalence, i.e. $f \sim g \Leftrightarrow f(t) / g(t) \rightarrow 1$ as $t \rightarrow \infty$. Applying these to $u_{1}$ and $u_{2}$ in (3.3) would provide more precise upper bounds in $\left(U_{1}\right)$ and $\left(U_{2}\right)$. For similar stronger bounds in $\left(L_{1}\right)$ and $\left(L_{2}\right)$ however, one would need strong large deviation results for Poisson processes.

3. Dropping the boundedness of the state space of $\mathcal{X}$, i.e. considering $s_{\alpha}=\infty$, (3.1) holds letting $\hat{\alpha}=\sup _{x \in \mathbb{R}_{+}} \frac{1}{x} \alpha(x)$ and so does 1. as well as $\left(U_{1}\right)$. Also, $\left(L_{1}\right)$ and $\left(L_{2}\right)$ still apply, starting $\mathcal{W}$ at $W_{0}:=\min \left\{1, X_{0}\right\}$. But we need boundedness from below of $\mathcal{Y}$ to prove (3.3) and the finiteness of $\mathbb{E}\left[S \mid Y_{0}=y\right]$ and thus lose $\left(U_{2}\right)$ as well as the limit results of 2.

4. Whenever $\left(X_{t}\right)$ is a $p$-jump process with drift $\alpha$, the process of the $k$ th powers, $\left(X_{t}^{k}\right)$, is a $p^{k}$-jump process with drift $\alpha_{k}: x \mapsto k x^{1-\frac{1}{k}} \alpha\left(x^{\frac{1}{k}}\right)$ holding $\hat{\alpha}_{k}=k \hat{\alpha}$ as well as $\left(\alpha_{k}\right)_{0}^{\prime}=k \alpha_{0}^{\prime}$. Hence, it would suffice to prove $\left(U_{1}\right)$ and $\left(U_{2}\right)$ for $k=1$. For $\left(L_{1}\right)$ and $\left(L_{2}\right)$ however, the lower bound on $\alpha$ only implies that $\alpha_{k}(x) \geq k \delta x-k \vartheta x^{1+\frac{1}{k}}$, such that for $k>1$ we can not use the process $\mathcal{W}$ in (3.4) as a lower bound process and the proof fails.

\subsection{Proof of Corollary 2.4}

Proof. The concavity of $\alpha$ implies that $x_{\alpha}$ equates to $x_{\alpha}$ and $s_{\alpha}$ from Theorem 1 Noting that, if $I=\mathbb{R}^{+}$and $s=\max \left\{x_{\alpha}, X_{0}\right\}, \alpha^{\prime}(s)>-\infty$, by the same scaling argument as in the beginning of the proof of Theorem 1 we can assume that $I=[0,1]$.

We start with $p \in(0,1)$ : Since $\alpha$ is concave, $x \mapsto \frac{\alpha(x)-\alpha(y)}{x-y}$ decreases on $(y, 1]$ and $y \mapsto \frac{\alpha(x)-\alpha(y)}{x-y}$ decreases on $(0, x)$. Thus, by the assertions, $\left|\frac{\alpha(x)-\alpha(y)}{x-y}\right|$ is bounded on each interval $[\varepsilon, 1]$ and bounded on $[0,1]$, if $\alpha_{0}^{\prime}<\infty$. Hence, either $\left(C_{1}\right)$ or $\left(C_{2}\right)$ holds, $\alpha$ suffices the assertions of Theorem 1 and the existence of $\mathcal{X}$ follows and so does 3 by Theorem 12 .

Recall that $\alpha_{0}^{\prime}=\alpha^{\prime}(0)$, if $\alpha_{0}^{\prime}<\infty$. Now, for 1 . we want to apply $\left(L_{1}\right)$ and $\left(L_{2}\right)$ of Theorem 1 using $\delta=\alpha_{0}^{\prime}=\alpha^{\prime}(0)$. Let us first assume that $\alpha^{\prime \prime}(0)>-\infty$. Then, there is $\vartheta>0$ such that $\alpha^{\prime}(0) x-\vartheta x^{2} \leq \alpha(x)$ for all $x \in[0,1]$. (The Taylor expansion delivers the existence of a $\vartheta_{0}$ such that $\alpha^{\prime}(0) x-\vartheta_{0} x^{2} \leq \alpha(x)$ holds for $x$ in some interval $[0, \varepsilon)$. The boundedness of $\alpha$ ensures that we can choose $\vartheta_{1}$ such that $\alpha^{\prime}(0) x-\vartheta_{1} x^{2} \leq \alpha(x)$ holds for $x \geq \varepsilon$. Let $\vartheta=\max \left\{\vartheta_{0}, \vartheta_{1}\right\}$.)

On the other hand, if $\alpha^{\prime \prime}(0)=-\infty, \alpha$ has no parabolic lower bound as we need for $\left(L_{1}\right)$ and $\left(L_{2}\right)$ (e.g. for $\alpha(x):=a x-b x^{3 / 2}$ with $b \geq a>0$ ). We solve this by a coupling argument: Therefore, let $p$ be fixed and for all $n$ define

$$
\alpha_{n}(x)=\min \left\{\alpha(x), x \alpha\left(\frac{1}{n}\right)\right\},
$$

the minimum of $\alpha$ and the secant of $\alpha$ intersecting at 0 and $\frac{1}{n}$. Surely, the $\alpha_{n}$ satisfy the conditions of Theorem $1 \alpha_{n} \nearrow \alpha$ point-wise, $\alpha^{\prime \prime}(0)=0>-\infty$ and $\alpha_{n}(0)=0$ such that $\left(\alpha_{n}\right)_{0}^{\prime}=\alpha_{n}^{\prime}(0)$. Let $\mathcal{X}^{(n)}$ be processes with respective generators

$$
\mathcal{G}_{n} f(x)=f(p x)-f(x)+\alpha_{n}(x) f^{\prime}(x)
$$

coupled in such a way that they jump simultaneously with $\mathcal{X}$ and each started in $X_{0}$. Then, $X_{t}^{(n)} \leq X_{t}$ for all $n$ and thus

$$
\liminf _{t \rightarrow \infty} \frac{1}{t} \log \mathbb{E}\left[X_{t}^{k}\right] \geq \sup _{n} \liminf _{t \rightarrow \infty} \frac{1}{t} \log \mathbb{E}\left[\left(X_{t}^{(n)}\right)^{k}\right] .
$$

From the previous case, we obtain for every $n$

$$
\liminf _{t \rightarrow \infty} \frac{1}{t} \log \mathbb{E}\left[\left(X_{t}^{(n)}\right)^{k}\right] \geq \begin{cases}-\left(1-p^{k}-\alpha_{n}^{\prime}(0) k\right) & \text { if } \alpha_{n}^{\prime}(0) \leq p^{k} \log \frac{1}{p} \\ -\left(1-\frac{1}{\gamma_{n}}\left(1+\log \gamma_{n}\right)\right) & \text { if } p^{k} \log \frac{1}{p}<\alpha_{n}^{\prime}(0)<\log \frac{1}{p}\end{cases}
$$

where $\gamma_{n}=\log \frac{1}{p} / \alpha_{n}^{\prime}(0)$. Since $\alpha_{n}^{\prime}(0)=\alpha\left(\frac{1}{n}\right) /\left(\frac{1}{n}\right) \nearrow \alpha^{\prime}(0)$ and the bounds in either case increase in $\alpha_{n}^{\prime}(0)$, we obtain " $\leq$ " in 1 . Since $\alpha$ is concave, it satisfies $\alpha^{\prime}(0)=\hat{\alpha}$ and thus, $\left(U_{1}\right)$ and $\left(U_{2}\right)$ of 
Theorem 1 provide " $\geq "$ and 1 . follows.

For the case $\alpha_{0}^{\prime}=\log \frac{1}{p}$ consider a family $\left(\mathcal{X}^{(p)}\right)_{p \in(0,1)}$ of processes with respective generators as in (2.1) with $\alpha$ fixed and $p \mapsto X_{0}^{(p)}$ constant, coupled in such a way that the processes jump simultaneously. Then, for every $t$ the map $p \mapsto X_{t}^{(p)}$ is increasing. Since $p^{k} \log \frac{1}{p}<\alpha_{0}^{\prime}$ for $p$ near $p^{*}:=e^{-\alpha_{0}^{\prime}}$, we conclude from 1 . and the boundedness of the $\mathcal{X}^{(p)}$ that

$$
\begin{aligned}
0 & \geq \lim _{t \rightarrow \infty} \frac{1}{t} \log \mathbb{E}\left[\left(X_{t}^{\left(p^{*}\right)}\right)^{k}\right] \geq \sup _{p<p^{*}} \lim _{t \rightarrow \infty} \frac{1}{t} \log \mathbb{E}\left[\left(X_{t}^{(p)}\right)^{k}\right] \\
& =\sup _{p<p^{*}}-\left(1-\frac{\alpha_{0}^{\prime}}{\log \frac{1}{p}}\left(1-\log \left(\frac{\alpha_{0}^{\prime}}{\log \frac{1}{p}}\right)\right)\right)=0 .
\end{aligned}
$$

Since $x \mapsto \frac{1}{x} \alpha(x)=\frac{1}{x}(\alpha(x)-\alpha(0))+\frac{1}{x} \alpha(0)$ is decreasing, Theorem 12 implies

$$
\alpha_{0}^{\prime} \geq \mathbb{E}\left[\liminf _{t \rightarrow \infty} \frac{\alpha\left(X_{t}^{\left(p^{*}\right)}\right)}{X_{t}^{\left(p^{*}\right)}}\right] \geq \sup _{p>p^{*}} \mathbb{E}\left[\liminf _{t \rightarrow \infty} \frac{\alpha\left(X_{t}^{(p)}\right)}{X_{t}^{(p)}}\right]=\sup _{p>p^{*}} \log \frac{1}{p}=\alpha_{0}^{\prime}
$$

and hence almost surely

$$
\liminf _{t \rightarrow \infty} \frac{\alpha\left(X_{t}^{\left(p^{*}\right)}\right)}{X_{t}^{\left(p^{*}\right)}}=\lim _{t \rightarrow \infty} \frac{\alpha\left(X_{t}^{\left(p^{*}\right)}\right)}{X_{t}^{\left(p^{*}\right)}}=\alpha_{0}^{\prime} .
$$

Since $x \mapsto \alpha(x) / x$ is decreasing, $\lim \sup _{t} X_{t}^{\left(p^{*}\right)}$ has to be bounded by $m_{\alpha}:=\sup \{x \in[0,1]: \alpha(x)=$ $\left.x \alpha_{0}^{\prime}\right\}$. Finally, if $\alpha$ is strictly concave near $0, \alpha(x) / x$ strictly decreases near 0 and thus $m_{\alpha}=0$, which concludes the proof for $p \in(0,1)$.

Considering $p=1$, we can ignore the jumps and $\mathcal{X}$ becomes deterministic, i.e. the solution of $\dot{X}_{t}=\alpha\left(X_{t}\right)$. Then, it holds if $\alpha_{0}^{\prime}<0=\log \left(\frac{1}{p}\right)$ that

$$
\frac{1}{t}\left(\log \left(X_{t}\right)-\log \left(X_{0}\right)\right)=\frac{1}{t} \int_{0}^{t} \frac{\dot{X}_{s}}{X_{s}} d s=\frac{1}{t} \int_{0}^{t} \frac{\alpha\left(X_{s}\right)}{X_{s}} d s \leq \alpha_{0}^{\prime}<0 .
$$

Hence, $X_{t} \rightarrow 0, \alpha\left(X_{t}\right) / X_{t} \rightarrow \alpha_{0}^{\prime}$ and $\frac{1}{t} \log \left(X_{t}^{k}\right) \rightarrow k \alpha_{0}^{\prime}$, which aligns with 1. However, if $\alpha_{0}^{\prime}=$ 0 , since $\alpha$ is concave it is non-positive. Then, $\mathcal{X}$ is constant, if $\alpha\left(X_{0}\right)=0$, and it converges monotonically to $m_{\alpha}=\sup \{x \in[0,1]: \alpha(x)=0\}$ if $X_{0}>m_{\alpha}$. Thus, $\lim _{t \rightarrow \infty} \frac{1}{t} \log \left(X_{t}\right)=$ $\lim _{t \rightarrow \infty} \alpha\left(X_{t}\right) / X_{t}=0$ giving us 2. Lastly, in the case of $\alpha_{0}^{\prime}>0, \mathcal{X}$ will either grow towards $x_{\alpha}$ if started below, i.e. $X_{0} \in\left(0, x_{\alpha}\right)$, or fall towards it if started above. Either way, $X_{t} \rightarrow x_{\alpha}$, $\alpha\left(X_{t}\right) \rightarrow 0$ and we obtain 3 .

Choosing $p=0, \mathcal{X}$ would jump to 0 after an exponentially distributed time $T$ with mean 1 and stay there indefinitely. Thus, we can write $X_{t}=Y_{t} \cdot \mathbb{1}_{\{T>t\}}$, where $\left(Y_{t}\right)$ is the deterministic process arising for $p=1$ discussed above. Then clearly, $\mathcal{X}$ will always converge to 0 almost surely and

$$
-\frac{1}{t} \log \left(\mathbb{E}\left[X_{t}^{k}\right]\right)=-\frac{1}{t} \log \mathbb{P}(T>t)-\frac{k}{t} \log \left(Y_{t}\right) \stackrel{t \rightarrow \infty}{\longrightarrow} 1+\max \left\{0,-k \alpha_{0}^{\prime}\right\} .
$$

\section{Branching processes with binomial disasters}

In the following subsections, we borrow ideas from the notion of duality of Markov processes; see Chapter 4.4 in Ethier and Kurtz (1986).

Recall that two Markov processes $\mathcal{Z}=\left(Z_{t}\right)_{t \geq 0}$ and $\mathcal{X}=\left(X_{t}\right)_{t \geq 0}$ with state spaces $E$ and $E^{\prime}$ are called dual with respect to the function $H: E \times E^{\prime} \rightarrow \mathbb{R}$ if

$$
\mathbb{E}\left[H\left(Z_{t}, x\right) \mid Z_{0}=z\right]=\mathbb{E}\left[H\left(z, X_{t}\right) \mid X_{0}=x\right]
$$


for all $z \in E, x \in E^{\prime}$. When one is interested in the process $\mathcal{Z}$, this relationship is most helpful if the process $\mathcal{X}$ is easier to analyse than the process $\mathcal{Z}$. Moreover, frequently, the set of functions $\left\{H(\cdot, x): x \in E^{\prime}\right\}$ is separating on $E$ such that the left hand side of (D) determines the distribution of $Z_{t}$. In this case, the distribution of the simpler process $\mathcal{X}$ determines via (D) the distribution of $\mathcal{Z}$, so analysing $\mathcal{Z}$ becomes feasible.

There is no straightforward way how to find dual processes, but they arise frequently in the literature; see Jansen and Kurt (2014) for a survey. Examples span reflected and absorbed Brownian motion, interacting particle models such as the voter model and the contact process, as well as branching processes.

A simple way to verify (D) for homogeneous $\mathcal{Z}$ and $\mathcal{X}$, is to show that

$$
\left.\frac{\partial}{\partial t} \mathbb{E}\left[H\left(Z_{t}, x\right) \mid Z_{0}=z\right]\right|_{t=0}=\left.\frac{\partial}{\partial t} \mathbb{E}\left[H\left(z, X_{t}\right) \mid X_{0}=x\right]\right|_{t=0}
$$

for all $z$ and $x$, since then both sides of (D) follow the same evolution.

\subsection{Proof of Theorem 2}

In this section we will discuss branching processes of the form of Definition 2.5. Hence, let $\mathcal{Z}:=\mathcal{Z}_{\lambda, q, \kappa, p}^{h}$, where $\lambda \in(0, \infty)$ is the death-rate, $q=\left(q_{k}\right)_{k \geq 0}$ the offspring distribution on $\mathbb{N}_{0}, p \in(0,1)$ the survival probability of the disasters that occur at the jump times of $\left(D_{t}\right)_{t \geq 0}$, a Poisson process with rate $\kappa>0$. Moreover, let $h:[0,1] \rightarrow[0,1], x \mapsto \sum_{k>0} q_{k} x^{k}$ be the probability generating function of the offspring distribution. We start with establishing a suitable duality for $\kappa=1$. The general case will follow by a rescaling argument.

Lemma 4.1. Let $p \in[0,1], \kappa=1$ and $\left(X_{t}\right)$ be a $p$-jump process with drift $x \mapsto \lambda(1-x-h(1-x))$, having the generator

$$
\mathcal{G}_{\mathcal{X}} f(x)=f(p x)-f(x)+\lambda(1-x-h(1-x)) f^{\prime}(x)
$$

for $f \in \mathcal{C}_{b}^{1}([0,1])$. Then, the duality relation

$$
\mathbb{E}\left[\left(1-X_{t}\right)^{z} \mid X_{0}=x\right]=\mathbb{E}\left[(1-x)^{Z_{t}} \mid Z_{0}=z\right]
$$

holds for every $x \in[0,1], z \in \mathbb{N}_{0}$ and $t \geq 0$.

Proof. Recalling the generator of $\mathcal{Z}$ from Definition 2.5. we obtain for $x \in[0,1], z \in \mathbb{N}_{0}$ and $H(x, z):=(1-x)^{z}$ that

$$
\begin{aligned}
\left(\mathcal{G}_{\mathcal{Z}}\right. & H(x, \cdot))(z) \\
& =\lambda z(1-x)^{z-1} \sum_{k \geq 0} q_{k}\left((1-x)^{k}-(1-x)\right)+\sum_{\ell=0}^{z}\left(\begin{array}{l}
z \\
\ell
\end{array}\right)(p(1-x))^{\ell}(1-p)^{z-\ell}-(1-x)^{z} \\
& =-\frac{\partial}{\partial x}(1-x)^{z} \cdot \lambda(h(1-x)-(1-x))+(p(1-x)+(1-p))^{z}-(1-x)^{z} \\
& =\left(\mathcal{G}_{\mathcal{X}} H(\cdot, z)\right)(x),
\end{aligned}
$$

which resembles $\left(\mathrm{D}^{2}\right)$. Hence, $(\mathrm{D})$ gives us the desired relation.

Now, we apply Corollary 2.4 to the dual process $\mathcal{X}$, followed by the proof of Theorem 2

Lemma 4.2. For $p \in[0,1]$, such a process $\mathcal{X}$ in Lemma 4.1] exists and satisfies

1. if $p=0$ or $h^{\prime}(1)<1+\frac{1}{\lambda} \log \frac{1}{p}, X_{t} \stackrel{t \rightarrow \infty}{\longrightarrow} 0$ almost surely. Also, for $k \geq 1$

$$
\lim _{t \rightarrow \infty}-\frac{1}{t} \log \mathbb{E}\left[X_{t}^{k}\right]= \begin{cases}1+\max \left\{0,-k\left(h^{\prime}(1)-1\right)\right\} & \text { if } p=0, \\ \left.1-p^{k}-k \lambda\left(h^{\prime}(1)-1\right)\right) & \text { if } h^{\prime}(1) \leq 1+\frac{p^{k}}{\lambda} \log \frac{1}{p}, \\ 1-\frac{1}{\gamma}(1+\log \gamma) & \text { otherwise, }\end{cases}
$$

where $\gamma=\frac{1}{\lambda} \log \frac{1}{p} /\left(h^{\prime}(1)-1\right)$. 
2. if $h^{\prime}(1)=1+\frac{1}{\lambda} \log \frac{1}{p}, X_{t} \stackrel{t \rightarrow \infty}{\longrightarrow} 0$ almost surely, while $\frac{1}{t} \log \mathbb{E}\left[X_{t}^{k}\right] \stackrel{t \rightarrow \infty}{\longrightarrow} 0$ for all $k$.

3. if $h^{\prime}(1) \in\left(1+\frac{1}{\lambda} \log \frac{1}{p}, \infty\right]$, letting $x_{*}$ be the smallest fixed point of $h, \mathcal{X}$ converges weakly to a random variable $X_{\infty}$ on $\left(0,1-x_{*}\right]$ that satisfies $\mathbb{E}\left[X_{\infty}^{-1}\left(1-h\left(1-X_{\infty}\right)\right)\right]=1+\frac{1}{\lambda} \log \frac{1}{p}$ and for $k \geq 1$

$$
\mathbb{E}\left[X_{\infty}^{k}\right]=\frac{\lambda k}{\lambda k+1-p^{k}} \mathbb{E}\left[X_{\infty}^{k-1}\left(1-h\left(1-X_{\infty}\right)\right)\right]
$$

Proof. Since $h$ is a convex function, $\alpha: x \mapsto \lambda(1-x-h(1-x))$ is concave. Also, $\alpha(0)=$ $\lambda(1-h(1))=0, \alpha(1)=-\lambda q_{0} \leq 0, \alpha_{0}^{\prime}=\alpha^{\prime}(0)=\lambda\left(h^{\prime}(1)-1\right) \in(-\lambda, \infty]$ and $\alpha^{\prime}(1)=\lambda\left(h^{\prime}(0)-1\right)=$ $\lambda\left(q_{1}-1\right) \geq-\lambda>-\infty$. Hence, considering that $\alpha^{\prime}(0) \geq p^{k} \log \frac{1}{p}$ iff $h^{\prime}(1) \leq 1+\frac{p^{k}}{\lambda} \log \frac{1}{p}$ for all $k \geq 1$, Corollary 2.4 implies 1. For 3., noting that $\alpha(x)>0$ only if $0<x<1-x_{*}=x_{\alpha}$, only (4.3) remains to be shown. Here, Corollary 2.43 gives us for $k \geq 1$

$$
\mathbb{E}\left[X_{\infty}^{k}\right]=\frac{\lambda k}{1-p^{k}}\left(-\mathbb{E}\left[X_{\infty}^{k}\right]+\mathbb{E}\left[X_{\infty}^{k-1}\left(1-h\left(1-X_{\infty}\right)\right)\right]\right)
$$

and (4.3) follows. Finally, if $h^{\prime}(1)=1+\frac{1}{\lambda} \log \frac{1}{p}>1, h$ is strictly convex and thus, $\alpha$ is strictly concave, which gives us 2 .

Proof of Theorem Q First, let the theorem hold for $\kappa=1$ and for arbitrary $\kappa>0$ consider the process $\mathcal{Z}^{*}:=\mathcal{Z}_{\lambda / \kappa, q, 1, p}^{h}$. Then, $\left(Z_{t}\right)_{t}:=\left(Z_{\kappa t}^{*}\right)_{t}$ defines a $\mathcal{Z}_{\lambda, q, \kappa, p}^{h}$-process and we obtain

$$
\lim _{t \rightarrow \infty}-\frac{1}{t} \log \mathbb{P}\left(Z_{t}>0\right)=\kappa \cdot \lim _{s \rightarrow \infty}-\frac{1}{s} \log \mathbb{P}\left(Z_{s}^{*}>0\right),
$$

which shows 1. and 2. Since $\lim _{t} Z_{t}=\lim _{t} Z_{t}^{*}$ almost surely, 3. follows, where we obtain (2.3) by substitution.

Hence, without loss of generality let $\kappa=1$. Then, letting $Z_{0}=k$ and $X_{0}=1$, Lemma 4.1 implies,

$$
\mathbb{P}\left(Z_{t}>0\right)=1-\mathbb{E}\left[0^{Z_{t}}\right]=1-\mathbb{E}\left[\left(1-X_{t}\right)^{k}\right]=\sum_{\ell=1}^{k}\left(\begin{array}{l}
k \\
\ell
\end{array}\right)(-1)^{\ell+1} \mathbb{E}\left[X_{t}^{\ell}\right] .
$$

Considering that $X_{t} \in[0,1]$, we obtain from Bernoulli's inequality

$$
\mathbb{E}\left[X_{t}\right] \leq 1-\mathbb{E}\left[\left(1-X_{t}\right)^{k}\right]=\mathbb{P}\left(Z_{t}>0\right) \leq k \mathbb{E}\left[X_{t}\right] .
$$

Thus, noting that $h^{\prime}(1)=\mu$ and $\gamma=1 / \nu$, for $\mu \leq 1+\frac{1}{\lambda} \log \frac{1}{p}$ (i.e. $\nu \leq 1$ ) Lemma 4.2 1 and 4.2.2 show that

$$
\lim _{t \rightarrow \infty}-\frac{1}{t} \log \mathbb{P}\left(Z_{t}>0\right)= \begin{cases}1+\max \left\{0,-k\left(h^{\prime}(1)-1\right)\right\} & \text { if } p=0, \\ 1-p-\lambda(\mu-1) & \text { if } \nu \leq p, \\ 1-\nu\left(1+\log \frac{1}{\nu}\right) & \text { if } p<\nu \leq 1\end{cases}
$$

Additionally, considering the boundedness and thus the $\mathcal{L}^{1}$-convergence of $\left(X_{t}\right)_{t}$, we get from (4.5) that $Z_{t}$ converges to 0 in probability. Since this implies almost sure convergence of a subsequence and 0 is an absorbing state, we have $Z_{t} \rightarrow 0$ almost surely.

For 2., noting that $\mathbb{P}\left(Z_{t} \stackrel{t \rightarrow \infty}{\longrightarrow} 0 \mid Z_{s}\right) \geq \frac{1}{1+\lambda}(1-p)^{Z_{s}}$, i.e. the probability that the next event after $s$ is a disaster that kills all, we obtain

$$
\limsup _{s \rightarrow \infty} \mathbb{P}\left(Z_{t} \stackrel{t \rightarrow \infty}{\longrightarrow} 0 \mid \sigma\left(Z_{r} ; r \leq s\right)\right) \geq \frac{1}{1+\lambda} \limsup _{s \rightarrow \infty}(1-p)^{Z_{s}}=\frac{1}{1+\lambda}(1-p)^{\liminf _{s \rightarrow \infty} Z_{s}} .
$$

Thus, Lemma 3.1 of (Kaplan et al., 1975, p.54) concludes

$$
\mathbb{P}\left(Z_{t} \stackrel{t \rightarrow \infty}{\longrightarrow} 0\right)+\mathbb{P}\left(Z_{t} \stackrel{t \rightarrow \infty}{\longrightarrow} \infty\right)=1 .
$$


Furthermore, for $\mu>1+\frac{1}{\lambda} \log \frac{1}{p}$ Lemma 4.23 shows stationarity of the distribution of $X_{\infty}$ and hence independence of $X_{0}$. Thus, using that 0 is an absorbing state and $\left\{Z_{s}=0\right\} \subset\left\{Z_{t}=0\right\}$ for $s \leq t$, we obtain from (4.4) that

$$
\mathbb{P}\left(\lim _{t \rightarrow \infty} Z_{t}=0\right)=\mathbb{P}\left(\bigcup_{t>0}\left\{Z_{t}=0\right\}\right)=\lim _{t \rightarrow \infty} \mathbb{P}\left(Z_{t}=0\right)=\mathbb{E}\left[\left(1-X_{\infty}\right)^{k}\right] .
$$

\subsection{Preparation: Regular Variation}

In this subsection, using results of chapter VIII.9 of Feller (1971) and Seneta (1976), we will arrange the tools regarding regularly varying functions needed for the proof of Theorem 3 . However, we need to establish some additional notation first:

\section{Remark 4.3.}

1. We will make use of the Bachmann-Landau notation: For a function $g: \mathbb{R}_{+} \rightarrow[0, \infty)$, let

$$
\begin{aligned}
o(g) & :=\left\{f: \mathbb{R}_{+} \rightarrow \mathbb{R}_{+} \mid \limsup _{t \rightarrow \infty} \frac{f(t)}{g(t)}=0\right\}, \\
O(g) & :=\left\{f: \mathbb{R}_{+} \rightarrow \mathbb{R}_{+} \mid \limsup _{t \rightarrow \infty} \frac{f(t)}{g(t)}<\infty\right\}, \\
\Omega(g) & :=\left\{f: \mathbb{R}_{+} \rightarrow \mathbb{R}_{+} \mid g \in O(f)\right\} .
\end{aligned}
$$

2. We define the relation of asymptotic equivalence for functions $f, g: \mathbb{R}_{+} \rightarrow \mathbb{R}$ by

$$
f \stackrel{t \rightarrow \infty}{\sim} g \quad \Leftrightarrow \quad \lim _{t \rightarrow \infty} \frac{f(t)}{g(t)}=1 .
$$

Often, when the running variable is either obvious or $t$, we will just write $f \sim g$.

Definition 4.4. A function $f: \mathbb{R}_{+} \rightarrow \mathbb{R}_{+}$is called regularly varying with exponent $\beta \in \mathbb{R}$, if for every $x>0$

$$
\frac{f(x t)}{f(t)} \stackrel{t \rightarrow \infty}{\longrightarrow} x^{\beta}
$$

holds. A slowly varying function is a regularly varying function with exponent 0.

Lemma 4.5. Let $f: \mathbb{R}_{+} \rightarrow \mathbb{R}_{+}$regularly varying with exponent $\beta \in \mathbb{R}$ and $F(t):=\int_{0}^{t} f(x) d x$.

1. $F$ is regularly varying with exponent $\max \{\beta+1,0\}$ and for $t \rightarrow \infty$, if

(a) $\beta>-1$, then $F(t) \sim t f(t)(\beta+1)^{-1}$.

(b) $\beta=-1$, then $F(t) \in \Omega(1) \cap O\left(t^{\varepsilon}\right)$ for all $\varepsilon>0$.

(c) $\beta<-1$, then $F(t) \rightarrow c<\infty$.

2. Let $\left(t_{n}\right) \subset \mathbb{R}_{+}$such that $t_{n} \stackrel{n \rightarrow \infty}{\longrightarrow} \infty$ and $t_{n+1} / t_{n} \stackrel{n \rightarrow \infty}{\longrightarrow} 1$. Then,

$$
\frac{F\left(t_{n+1}\right)}{F\left(t_{n}\right)} \stackrel{n \rightarrow \infty}{\longrightarrow} 1
$$

3. There are functions $a$ and $\varepsilon$ such that $a(t) \stackrel{t \rightarrow \infty}{\longrightarrow} c \in \mathbb{R}_{+}, \varepsilon(t) \stackrel{t \rightarrow \infty}{\longrightarrow} 0$ and

$$
f(t)=t^{\beta} a(t) \exp \left(\int_{1}^{t} \frac{\varepsilon(y)}{y} d y\right) .
$$


4. For each $\alpha>0, f \in O\left(t^{\beta+\alpha}\right) \cap \Omega\left(t^{\beta-\alpha}\right)$.

Proof. 3. and 4. follow from Seneta (1976), Theorem 1.1 on page 2 and Proposition $1^{0}$ on page 18 respectively, while 1 . is a consequence of 4. and exercises 2.1, 2.2 and 2.3 on (Seneta, 1976, p.86). (A proof of these exercises is given by Theorem 1 in Feller, 1971, p.281.)

Finally, by $1 . F$ is regularly varying with exponent $\beta^{\prime} \geq 0$. Applying 3 . we see that there are functions $A$ and $\mathcal{E}$ with $\lim _{t \rightarrow \infty} A(t)=c \in(0, \infty)$ and $\lim _{t \rightarrow \infty} \mathcal{E}(t)=0$ such that

$$
\frac{F\left(t_{n+1}\right)}{F\left(t_{n}\right)}=\left(\frac{t_{n+1}}{t_{n}}\right)^{\beta^{\prime}} \cdot \frac{A\left(t_{n+1}\right)}{A\left(t_{n}\right)} \cdot \exp \left(\int_{t_{n}}^{t_{n+1}} \frac{\mathcal{E}(y)}{y} d y\right) .
$$

Now, the first two factors converge to 1 , while the integral in the exponent is bounded by $\mid t_{n+1}-$ $t_{n}\left|\cdot \frac{1}{t_{n}} \sup _{y \in\left[t_{n}, t_{n+1}\right]}\right| \mathcal{E}(y) \mid \longrightarrow_{n \rightarrow \infty} 0$.

The following Theorem is needed in the proof of Theorem 3 to build a bridge between the asymptotics of the deterministic rate functions and the almost sure asymptotics of the process $\left(L_{t}\right)$ from Lemma 4.9] which is key to the computation of the survival probability in the inhomogeneous case.

Theorem 4.6. Let $\left(D_{t}\right)_{t \geq 0}$ be an inhomogeneous Poisson process with right continuous ratefunction $\kappa$ with left limits, $\Lambda(t):=\int_{0}^{t} \kappa_{s} d s, \Lambda^{-1}(t):=\inf \{s>0: \Lambda(s)>t\}$ and $f: \mathbb{R}_{+} \rightarrow \mathbb{R}_{+}$ such that $f\left(\Lambda^{-1}(\cdot)\right)$ is regularly varying with exponent $\beta$.

1. If $\Lambda(t) \stackrel{t \rightarrow \infty}{\longrightarrow} \Lambda(\infty)<\infty$ or $\beta<-1$, then $\int_{0}^{t} f(s) d D_{s}$ has an almost surely finite limit.

2. If $\Lambda(t) \stackrel{t \rightarrow \infty}{\longrightarrow} \infty$ and $\beta>-1$,

$$
\int_{0}^{t} f(s) d D_{s} \sim \int_{0}^{t} f(s) \kappa_{s} d s
$$

holds almost surely and in $\mathcal{L}^{2}$.

3. If $\beta=-1$, for arbitrary $\alpha>0$ it holds $\frac{\int_{0}^{t} f(s) d D_{s}}{\int_{0}^{t} f(s) \kappa_{s} d s} \in O\left(t^{\alpha}\right) \cap \Omega\left(t^{-\alpha}\right)$ almost surely.

Proof. First note that there is a unit-rate Poisson process, which we denote by $\left(P_{t}\right)$ and its jump times by $\left(\sigma_{k}\right)_{k}$, such that $D_{t}=P_{\Lambda(t)}$ for all $t \geq 0$ and the jump times of $\left(D_{t}\right)$ satisfy $\tau_{k}=\Lambda^{-1}\left(\sigma_{k}\right)$. Then, supposing that 2. holds for $\kappa \equiv 1$, the general case follows as

$$
\int_{0}^{t} f(s) d D_{s}=\sum_{k=1}^{D_{t}} f\left(\tau_{k}\right)=\sum_{k=1}^{P_{\Lambda(t)}} f\left(\Lambda^{-1}\left(\sigma_{k}\right)\right) \sim \int_{0}^{\Lambda(t)} f\left(\Lambda^{-1}(s)\right) d s=\int_{0}^{t} f(s) \kappa_{s} d s .
$$

Thus, without loss of generality, let $\kappa \equiv 1,\left(D_{t}\right)=\left(P_{t}\right)$ and $f$ regularly varying with exponent $\beta>-1$. Letting $F(t)=\int_{0}^{t} f(x) d x$, it remains to be shown that

$$
Y_{t}:=\frac{1}{F(t)} \sum_{k=1}^{D_{t}} f\left(\tau_{k}\right) \stackrel{t \rightarrow \infty}{\longrightarrow} 1
$$

almost surely and in $\mathcal{L}^{2}$. Starting with the $\mathcal{L}^{2}$-convergence, we recall that on the event $\left\{D_{t}=n\right\}$, the jump times $\left(\tau_{1}, \ldots, \tau_{n}\right)$ are equal in distribution to $\left(U_{(1)}^{t}, U_{(2)}^{t}, \ldots, U_{(n)}^{t}\right)$, the order statistic of $n$ iid variables $\left(U_{1}^{t}, \ldots, U_{n}^{t}\right)$, uniformly distributed on $[0, t]$. We obtain

$$
\begin{aligned}
\mathbb{E}\left[\sum_{k=1}^{D_{t}} f\left(\tau_{k}\right)\right] & =\mathbb{E}\left[\sum_{k=1}^{D_{t}} \mathbb{E}\left[f\left(\tau_{k}\right) \mid D_{t}\right]\right]=\mathbb{E}\left[\sum_{k=1}^{D_{t}} \mathbb{E}\left[f\left(U_{k}^{t}\right) \mid D_{t}\right]\right] \\
& =t \mathbb{E}\left[f\left(U_{1}^{t}\right)\right]=t \cdot \frac{1}{t} \int_{0}^{t} f(s) d s=F(t) .
\end{aligned}
$$


Hence, $\mathbb{E}\left[Y_{t}\right]=1$ and we compute

$$
\begin{aligned}
\left\|Y_{t}-1\right\|_{\mathcal{L}^{2}} & =\operatorname{Var}\left[\frac{1}{F(t)} \sum_{k=1}^{D_{t}} f\left(\tau_{k}\right)\right]=\frac{1}{F(t)^{2}}\left(\operatorname{Var}\left[\sum_{k=1}^{D_{t}} \mathbb{E}\left[f\left(U_{k}^{t}\right) \mid D_{t}\right]\right]+\mathbb{E}\left[\sum_{k=1}^{D_{t}} \operatorname{Var}\left[f\left(U_{k}^{t}\right) \mid D_{t}\right]\right]\right) \\
& =\frac{1}{F(t)^{2}}\left(\operatorname{Var}\left[D_{t} \mathbb{E}\left[f\left(U_{1}^{t}\right)\right]\right]+\mathbb{E}\left[D_{t} \operatorname{Var}\left[f\left(U_{1}^{t}\right)\right]\right]\right)=\frac{1}{F(t)^{2}}\left(t \mathbb{E}\left[f\left(U_{1}^{t}\right)\right]^{2}+t \operatorname{Var}\left[f\left(U_{1}^{t}\right)\right]\right) \\
& =\frac{t \mathbb{E}\left[f\left(U_{1}^{t}\right)^{2}\right]}{F(t)^{2}}=\frac{\int_{0}^{t} f(x)^{2} d x}{\left(\int_{0}^{t} f(x) d x\right)^{2}} .
\end{aligned}
$$

Since $f^{2}$ is regularly varying with exponent $2 \beta$, we obtain from Lemma 4.5. 1 and 4.5 .4 for

- $\beta>-\frac{1}{2}$, that $\left\|Y_{t}-1\right\|_{\mathcal{L}^{2}} \sim \frac{1}{t} \cdot \frac{(\beta+1)^{2}}{2 \beta+1}$.

- $\beta=-\frac{1}{2}$, some slowly varying function $\ell$ and arbitrary $\varepsilon>0$ that

$$
\left\|Y_{t}-1\right\|_{\mathcal{L}^{2}}=\frac{\ell(t)}{t} \in O\left(t^{-1+\varepsilon}\right) .
$$

- $\beta \in\left(-1,-\frac{1}{2}\right)$, the numerator converges to a constant and the denominator converges to $\infty$.

Either way, the $\mathcal{L}^{2}$ convergence follows.

For the almost sure convergence first note that there is a subsequence $\left(t_{n}\right)_{n}$ with $t_{n} \nearrow \infty$ as well as $\lim _{n \rightarrow \infty} Y_{t_{n}}=1$ and hence, $\liminf _{t} Y_{t} \leq 1 \leq \limsup _{t} Y_{t}$ almost surely. Noting that $\left(Y_{t}\right)$ is a piecewise deterministic process, jumping upwards and between jumps decreasing continuously, the maximum and minimum on the $n$th deterministic piece of the path respectively are given by

$$
Y_{n}^{+}:=Y_{\tau_{n}}=\frac{1}{F\left(\tau_{n}\right)} \sum_{k=1}^{n} f\left(\tau_{k}\right) \quad \text { and } \quad Y_{n}^{-}:=Y_{\tau_{n+1}-}=\frac{1}{F\left(\tau_{n+1}\right)} \sum_{k=1}^{n} f\left(\tau_{k}\right)
$$

and we obtain for every $t$ that $Y_{D_{t}}^{-} \leq Y_{t} \leq Y_{D_{t}}^{+}$. Also, we deduce from Lemma 4.5, 2 that

$$
\frac{Y_{n}^{+}}{Y_{n}^{-}}=\frac{F\left(\tau_{n+1}\right)}{F\left(\tau_{n}\right)} \stackrel{n \rightarrow \infty}{\longrightarrow} 1
$$

almost surely, considering that $\tau_{n+1} / \tau_{n} \stackrel{n \rightarrow \infty}{\longrightarrow} 1$. Since the values of the local extrema of $\left(Y_{t}\right)_{t}$ are given by $Y^{+}$and $Y^{-}$, it follows that

$$
\liminf _{n \rightarrow \infty} Y_{n}^{+}=\liminf _{n \rightarrow \infty} Y_{n}^{-}=\liminf _{t \rightarrow \infty} Y_{t} \leq 1 \leq \limsup _{t \rightarrow \infty} Y_{t}=\limsup _{n \rightarrow \infty} Y_{n}^{+} .
$$

Hence, it suffices to show that $Y_{n}^{+} \stackrel{n \rightarrow \infty}{\longrightarrow} 1$ almost surely. For this, let $h(n):=\min \{\sqrt{n}, \sqrt{F(n)}\}$ and decompose $Y_{n}^{+}$in the following way:

$$
Y_{n}^{+}=\frac{1}{F\left(\tau_{n}\right)} \sum_{k \leq h(n)} f\left(\tau_{k}\right)+\frac{1}{F\left(\tau_{n}\right)} \sum_{h(n)<k \leq n} \frac{1}{f\left(\tau_{k}\right)} .
$$

Considering that $A^{-}:=\inf _{n \geq 1} \frac{\tau_{n}}{n}>0$ and $A^{+}:=\sup _{n \geq 1} \frac{\tau_{n}}{n}<\infty$ almost surely, by Lemma 4.5 we obtain for the first part

$$
\begin{aligned}
\frac{1}{F\left(\tau_{n}\right)} \sum_{k \leq h(n)} f\left(\tau_{k}\right) & \sim \frac{\beta+1}{n} \sum_{k \leq h(n)}\left(\frac{\tau_{k}}{\tau_{n}}\right)^{\beta} \cdot \frac{a\left(\tau_{k}\right)}{a\left(\tau_{n}\right)} \cdot \exp \left(\int_{\tau_{k}}^{\tau_{n}} \frac{\varepsilon(y)}{y} d y\right) \\
& \leq \frac{C}{n} \cdot\left(\frac{A^{+}}{A^{-}}\right)^{|\beta|} \exp \left(\int_{0}^{n A^{+}} \frac{|\varepsilon(y)|}{y} d y\right) \cdot \sum_{k \leq h(n)}\left(\frac{k}{n}\right)^{\beta}
\end{aligned}
$$


where the constant $C$ arises from the boundedness of $a$. Now, for $\beta \geq 0$ the remaining sum is bounded above by $h(n) \leq \sqrt{n}$, while for $-1<\beta<0$ it holds for some slowly varying function $\ell$ that

$$
\sum_{k \leq h(n)}\left(\frac{k}{n}\right)^{\beta} \leq \sum_{k \leq h(n)} n^{|\beta|} \leq h(n) n^{|\beta|}=n^{|\beta|+\frac{1+\beta}{2}} \ell(n)=n^{\frac{1+|\beta|}{2}} \ell(n) .
$$

Thus, noting that $\frac{1+|\beta|}{2}<1$ and $\exp \left(\int_{0}^{\bullet} \frac{|\varepsilon(y)|}{y} d y\right)$ is slowly varying, it follows that

$$
\frac{1}{F\left(\tau_{n}\right)} \sum_{k \leq h(n)} f\left(\tau_{k}\right) \stackrel{n \rightarrow \infty}{\longrightarrow} 0
$$

almost surely. Hence, since $\tau_{n} \sim n$ almost surely and thus $F(n) \sim F\left(\tau_{n}\right)$ and $f\left(\tau_{k}\right) \sim f(k)$ by Lemma 4.5, 2, it follows from (4.7) and Lemma 4.5. 1(a)

$$
\begin{aligned}
Y_{n}^{+} & \sim \frac{F(n)}{F\left(\tau_{n}\right)} \cdot \frac{1}{F(n)} \sum_{h(n)<k \leq n} \frac{f\left(\tau_{k}\right)}{f(k)} \cdot f(k) \sim \frac{\beta+1}{n} \sum_{h(n)<k \leq n} \frac{f(k)}{f(n)} \\
& \sim \frac{\beta+1}{n} \sum_{h(n)<k \leq n}\left(\frac{k}{n}\right)^{\beta} \sim(\beta+1) \int_{h(n) / n}^{1} x^{\beta} d x \stackrel{n \rightarrow \infty}{\longrightarrow} 1
\end{aligned}
$$

and the proof of 2 . is done.

For 1. if $\Lambda(\infty)<\infty$, also $\lim _{t \rightarrow \infty} \int_{0}^{t} f(s) d D_{s}=\sum_{k=1}^{P_{\Lambda(\infty)}} f\left(\tau_{k}\right)$ is almost surely finite. Otherwise, we obtain from (4.6) and Lemma 4.51 that

$$
\mathbb{E}\left[\int_{0}^{t} f(s) d D_{s}\right]=\int_{0}^{t} f(s) \kappa_{s} d s \leq \int_{0}^{\infty} f(s) \kappa_{s} d s=\int_{0}^{\infty} f\left(\Lambda^{-1}(s)\right) d s<\infty,
$$

which also, by monotone convergence, implies the finiteness of $\int_{0}^{\infty} f(s) d D_{s}$ and 1 . is done.

Lastly, for 3. we conclude that for $\alpha>0, F(t):=\int_{0}^{t} f(s) \kappa_{s} d s$ and $Y_{t}:=\int_{0}^{t} f(s) d D_{s} / F(t)$

$$
0 \leq t^{-\alpha} Y_{t} \leq \frac{1}{F(t)} \int_{0}^{t} s^{-\alpha} f(s) d D_{s}
$$

Now, since $t \mapsto t^{-\alpha} f(t)$ is regularly varying with exponent $-1-\alpha<-1,1$. shows that the integral almost surely converges to some finite limit and hence, considering that $F$ is non-decreasing and non-negative, $\lim \sup _{t} t^{-\alpha} Y_{t}<\infty$ almost surely and $Y_{t} \in O\left(t^{\alpha}\right)$. Similarly, using 2.

$$
t^{\alpha} Y_{t} \geq \frac{1}{F(t)} \int_{0}^{t} s^{\alpha} f(s) d D_{s}
$$

which either converges to a positive constant, if $\Lambda(\infty)<\infty$, or is asymptotically equivalent to

$$
\frac{\int_{0}^{t} s^{\alpha} f(s) \kappa_{s} d s}{\int_{0}^{t} f(s) \kappa_{s} d s} \sim \frac{\int_{1}^{t} s^{\alpha} f(s) \kappa_{s} d s}{\int_{1}^{t} f(s) \kappa_{s} d s} \geq 1
$$

Either way, it follows that $\lim \sup _{t} t^{\alpha} Y_{t}>0$ and thus $Y_{t} \in \Omega\left(t^{-\alpha}\right)$.

Remark 4.7 (More precise asymptotics for $\beta=-1$ ). In the case $\beta=-1$ it follows from Lemma 4.5 that $F$ is slowly varying and thus, considering its monotonicity, lies in $O\left(t^{\varepsilon}\right) \cap \Omega(1)$ for all $\varepsilon>0$. As discussed in Polfeldt (1969) however, it is not always the case, that a regularly varying function with exponent -1 is integrable on $\mathbb{R}_{+}$. Supposing that $F(\infty)=\infty$, we obtain the $\mathcal{L}^{2}$-convergence in Theorem 4.6 analogously to the case $\beta \in\left(-1,-\frac{1}{2}\right)$, while the methods we used to obtain almost 
sure convergence fail for $\beta=-1$. Conversely, if $F(\infty)<\infty$, similarly to the proof of Theorem 4.6. 1 it follows that

$$
\lim _{t \rightarrow \infty} \mathbb{E}\left[\int_{0}^{t} f(s) d D_{s}\right]<\infty
$$

and the integral has a finite almost sure limit. Surely, Polfeldt (1969) can be used to specify the results for this critical case.

\subsection{Proof of Theorem 3}

In this section we generalise the findings of Corollary 2.7 to the time-inhomogeneous case. Recalling Definition 2.8, let $\mathcal{Z}=\mathcal{Z}_{b, d, \kappa, p}^{\text {in }}$ with birth, death and disaster rate functions $b, d$ and $\kappa$ respectively, and $p: \mathbb{R}_{+} \rightarrow[0,1]$ the survival probability function. Furthermore, let $\left(D_{t}\right)_{t}$ be the inhomogeneous Poisson process with rate $\kappa$ that counts the disasters up to time $t$. In what follows we will always assume $b, d$ and $\kappa$ to be right continuous with left limits and $p$ to be left continuous with right limits.

We start by computing the pgf of $\mathcal{Z}$ for $(1-p) \kappa \equiv 0$, i.e. without disasters, which will be generalised in Lemma 4.9

Lemma 4.8. Let $v(t):=\int_{0}^{t}\left(b_{y}-d_{y}\right) d y$ and $(1-p) \kappa \equiv 0$. Then, for $x \in[0,1], t \geq t_{0} \geq 0$ and $k \geq 0$ it holds that

$$
\mathbb{E}\left[(1-x)^{Z_{t}} \mid Z_{t_{0}}=k\right]=(1-s(t, x))^{k},
$$

where $s(t, 0)=0$ for all $t$ and $s(t, x)^{-1}=\frac{1}{x} e^{v\left(t_{0}\right)-v(t)}+e^{v\left(t_{0}\right)} \int_{t_{0}}^{t} b_{y} e^{-v(y)} d y$ for $x>0$.

Proof. Given that $Z_{t_{0}}=k, Z_{t}$ is equal in distribution to a sum of $k$ independent copies started in 1 at time $t_{0}$. Thus, $\mathbb{E}\left[(1-x)^{Z_{t}} \mid Z_{t_{0}}=k\right]=\mathbb{E}\left[(1-x)^{Z_{t}} \mid Z_{t_{0}}=1\right]^{k}$. Hence, without loss of generality we assume $k=1$. Now, considering Kendall (1948), where birth- and death-rate are denoted by $\lambda$ and $\mu$ respectively and $v$ is denoted by $-\rho$ (cf. (11)), by (9),(12) and (10b) we can compute for $z \in[0,1]$ that

$$
\begin{aligned}
\mathbb{E}\left[z^{Z_{t}} \mid Z_{0}=1\right] & =: \varphi(z, t)=\frac{1+e^{v(t)} \int_{0}^{t} b_{s} e^{-v(s)} d s-e^{v(t)}+\left(e^{v(t)}-e^{v(t)} \int_{0}^{t} b_{s} e^{-v(s)} d s\right) z}{1+\int_{0}^{t} b_{s} e^{-v(s)} d s-\int_{0}^{t} b_{s} e^{-v(s)} d s \cdot z} \\
& =1-\frac{e^{v(t)}-e^{v(t)} z}{1+\int_{0}^{t} b_{s} e^{-v(s)} d s \cdot(1-z)} .
\end{aligned}
$$

Substitution of $z=1-x$ and reducing the fraction by $x e^{v(t)}$ concludes the proof for $t_{0}=0$. The general case $t_{0} \geq 0$ is obtained considering a process $\mathcal{Z}^{*}$ with birth and death rates at time $s$ given by $b^{*}(s):=b_{t_{0}+s}$ and $d^{*}:=d_{t_{0}+s}$ respectively. Then, for $t \geq t_{0}$

$$
\mathbb{E}\left[(1-x)^{Z_{t}} \mid Z_{t_{0}}=1\right]=\mathbb{E}\left[(1-x)^{Z_{t-t_{0}}^{*}} \mid Z_{0}^{*}=1\right]=1-\frac{1}{\frac{1}{x} e^{-v_{t-t_{0}}^{*}+\int_{0}^{t-t_{0}} b^{*}(s) e^{-v_{s}^{*}} d s},}
$$

where

$$
v_{s}^{*}=\int_{0}^{s}\left(b^{*}(y)-d^{*}(y)\right) d y=\int_{t_{0}}^{t_{0}+s}\left(b_{y}-d_{y}\right) d y=v\left(t_{0}+s\right)-v\left(t_{0}\right)
$$

Substituting $y:=s+t_{0}$ in (4.8) and using (4.9) concludes the proof.

The following lemma generalises the result above to processes with disasters, i.e. $(1-p) \kappa \not \equiv 0$. It delivers a dual process $\mathcal{X}$ with respect to the pgf and thus corresponds to Lemma 4.1 in the proof of Theorem 2 
Lemma 4.9 (A stronger duality). Let $\log \frac{1}{0}=-\infty, 1 / 0=\infty$ and $1 / \infty=0$. Then, for $x \in[0,1]$, $k \geq 0$ and $\mathcal{D}_{\infty}:=\sigma\left(D_{s} ; s \geq 0\right)$, it holds that

$$
\mathbb{E}_{k}\left[(1-x)^{Z_{t}} \mid \mathcal{D}_{\infty}\right]=\left(1-X_{t}\right)^{k}
$$

for a piecewise deterministic process $\mathcal{X}=\left(X_{t}\right)_{t \geq 0}$ given by

$$
X_{t}^{-1}=\frac{1}{x} e^{-L_{t}}+\int_{0}^{t} e^{-L_{s}} b_{s} d s
$$

where $L_{t}=\int_{0}^{t}\left(b_{s}-d_{s}\right) d s-\int_{0}^{t} \log \left(\frac{1}{p_{s}}\right) d D_{s}$.

Proof. Let $t$ be fixed, $G_{t}(x):=\mathbb{E}_{k}\left[(1-x)^{Z_{t}} \mid \mathcal{D}_{\infty}\right], \tau_{0}:=0$ and $\tau_{1}, \tau_{2}, \ldots$ be the jump times of $\left(D_{t}\right)$, i.e. the disaster times of $\mathcal{Z}$. Note that the binomial disasters provide (with the left-side limit $\left.Z_{\tau_{n}-}:=\lim _{s \uparrow \tau_{n}} Z_{s}\right)$

$$
\mathbb{E}\left[(1-x)^{Z_{\tau_{n}}} \mid Z_{\tau_{n}-}=z\right]=\sum_{\ell=0}^{z}\left(\begin{array}{l}
z \\
\ell
\end{array}\right) p_{\tau_{n}}^{\ell}\left(1-p_{\tau_{n}}\right)^{z-\ell}(1-x)^{\ell}=\left(p_{\tau_{n}}(1-x)+\left(1-p_{\tau_{n}}\right)\right)^{z}=\left(1-p_{\tau_{n}} x\right)^{z} .
$$

Iterating this and Lemma 4.8, we obtain

$$
\begin{aligned}
& G_{t}(x)=\mathbb{E}\left[\mathbb{E}\left[(1-x)^{Z_{t}} \mid Z_{\tau_{D_{t}}}, \mathcal{D}_{\infty}\right] \mid \mathcal{D}_{\infty}\right]=\mathbb{E}\left[\left(1-s_{D_{t}}\right)^{Z_{\tau_{D_{t}}}} \mid \mathcal{D}_{\infty}\right] \\
& =G_{\tau_{D_{t}}}\left(s_{D_{t}}\right) \\
& \text { with } s_{D_{t}}^{-1}=\frac{e^{v\left(\tau_{D_{t}}\right)-v(t)}}{x}+e^{v\left(\tau_{D_{t}}\right)} \int_{\tau_{D_{t}}}^{t} b_{s} e^{-v(s)} d s \\
& =G_{\tau_{D_{t}}-}\left(p_{\tau_{D_{t}}} s_{D_{t}}\right)=\ldots \\
& =G_{\tau_{D_{t}-1}}\left(s_{D_{t}-1}\right) \\
& \text { with } s_{D_{t}-1}^{-1}=\frac{e^{v\left(\tau_{D_{t}-1}\right)-v\left(\tau_{D_{t}}\right)}}{p_{\tau_{D_{t}}} s_{D_{t}}}+e^{v\left(\tau_{D_{t}-1}\right)} \int_{\tau_{D_{t}-1}}^{\tau_{D_{t}}} b_{s} e^{-v(s)} d s \\
& =G_{\tau_{D_{t}-2}}\left(s_{D_{t}-2}\right) \\
& \text { with } s_{D_{t}-2}^{-1}=\frac{e^{v\left(\tau_{D_{t}-2}\right)-v\left(\tau_{D_{t}-1}\right)}}{p_{\tau_{D_{t}-1}} s_{D_{t}-1}}+e^{v\left(\tau_{D_{t}-2}\right)} \int_{\tau_{D_{t}-2}}^{\tau_{D_{t}-1}} b_{s} e^{-v(s)} d s \\
& =\ldots=G_{\tau_{0}}\left(s_{0}\right) \\
& \text { with } s_{0}=\frac{e^{v\left(\tau_{0}\right)-v\left(\tau_{1}\right)}}{p_{\tau_{1}} s_{1}}+e^{v\left(\tau_{0}\right)} \int_{\tau_{0}}^{\tau_{1}} b_{s} e^{-v(s)} d s \\
& =\left(1-s_{0}\right)^{k} \text {. }
\end{aligned}
$$


Now, solving the recursion,

$$
\begin{aligned}
s_{0}^{-1}=(\ldots( & \left.\frac{1}{x} e^{v\left(\tau_{D_{t}}\right)-v(t)}+e^{v\left(\tau_{D_{t}}\right)} \int_{\tau_{D_{t}}}^{t} b_{y} e^{-v(y)} d y\right) \\
& \left.\left.\cdot \frac{1}{p_{\tau_{D_{t}}}} e^{v\left(\tau_{D_{t}-1}\right)-v\left(\tau_{D_{t}}\right)}+e^{v\left(\tau_{D_{t}-1}\right)} \int_{\tau_{D_{t}-1}}^{\tau_{D_{t}}} b_{y} e^{-v(y)} d y\right) \cdots\right) \\
& \left.\cdot \frac{1}{p_{\tau_{1}}} e^{v\left(\tau_{0}\right)-v\left(\tau_{1}\right)}+e^{v\left(\tau_{0}\right)} \int_{\tau_{0}}^{\tau_{1}} b_{y} e^{-v(y)} d y\right) \\
=\frac{1}{x} e^{-v(t)} & \prod_{k=1}^{D_{t}} p_{\tau_{k}}^{-1}+\sum_{k=0}^{D_{t}} \prod_{\ell=1}^{k} p_{\tau_{\ell}}^{-1} \cdot e^{v\left(\tau_{0}\right)} \int_{\tau_{k}}^{\tau_{k+1} \wedge t} b_{y} e^{-v(y)} d y,
\end{aligned}
$$

where the empty product equals 1 . (Then, for $D_{t}=0$ and thus $t \leq \tau_{1}$, one obtains the deterministic dual from Lemma 4.8) Letting $\beta(t):=\int_{0}^{t} b_{s} e^{-v(s)} d s$ and considering that $v\left(\tau_{0}\right)=v(0)=0$, the sum equates to

$$
\sum_{k=0}^{D_{t}} \prod_{\ell=1}^{k} p_{\tau_{\ell}}^{-1}\left(\beta\left(\tau_{k+1} \wedge t\right)-\beta\left(\tau_{k}\right)\right)=\int_{0}^{t} \prod_{\ell=1}^{D_{s}} p_{\tau_{\ell}}^{-1} \cdot b_{s} e^{-v(s)} d s .
$$

With

$$
\prod_{k=1}^{D_{t}} p_{\tau_{k}}^{-1}=\exp \left(\sum_{s \leq t} \log \frac{1}{p_{s}} \cdot\left(D_{s}-D_{s-}\right)\right)=\exp \left(\int_{0}^{t} \log \frac{1}{p_{s}} d D_{s}\right),
$$

it is simple to deduce that $s_{0}$ equals $X_{t}$ from (4.10) and the proof is done.

\section{Remark 4.10.}

1. This Lemma holds for arbitrary counting processes $\left(D_{t}\right)_{t \geq 0}$. One might even consider a process with multiple jumps, e.g. $\mathbb{P}\left(\tau_{k}=0\right)>0$ for some $k$.

2. The process $\mathcal{X}$ here is not of the form required for Corollary 2.4 or Theorem 1 even if we choose constant $b, d, \kappa, p$ to obtain homogeneity: $X_{t}$ jumps from a state $1 /\left(\frac{a}{x}+b\right)$ to $1 /\left(\frac{a}{p x}+b\right)$, which is not a $p$-jump. However, in the homogeneous case, letting $b \equiv \vartheta>0$, $d \in \mathbb{R}_{+}, \delta:=b-d>0$ and $\kappa \equiv 1$, we can see that $X_{t}$ equates to $\bar{W}_{t}$, the time-reversal of $W_{t}$ in (3.4) we used in the proof of Theorem 1. Similarly one obtains that the (homogeneous) time-reversal $\bar{X}_{t}$ has the generator, for $f \in \mathcal{C}^{1}([0,1])$,

$$
\mathcal{G}_{\overline{\mathcal{X}}} f(x)=\kappa(f(p x)-f(x))+(b x(1-x)-d x) f^{\prime}(x) .
$$

3. The relationship between $\mathcal{X}$ and $\mathcal{Z}$ can be viewed as a stronger duality, since the duality relation (D), from the beginning of Section 4 , here does not only hold in expectation, but even in conditional expectation. (Taking expectation, (D) follows.)

Although we are not able to use Corollary 2.4 here, from the previous Lemma we immediately obtain the following

Proposition 4.11. Let $L_{t}$ as in Lemma 4.9 and $I_{t}:=\int_{0}^{t} e^{-L_{s}} b_{s} d s$. Supposing that $L_{t} \stackrel{t \rightarrow \infty}{\longrightarrow} L \in$ $[-\infty, \infty]$ almost surely and letting $I:=\lim _{t \rightarrow \infty} I_{t}$, there are 3 possible outcomes for the limit of 
$\mathbb{E}_{k}\left[(1-x)^{Z_{t}} \mid \mathcal{D}_{\infty}\right]:$

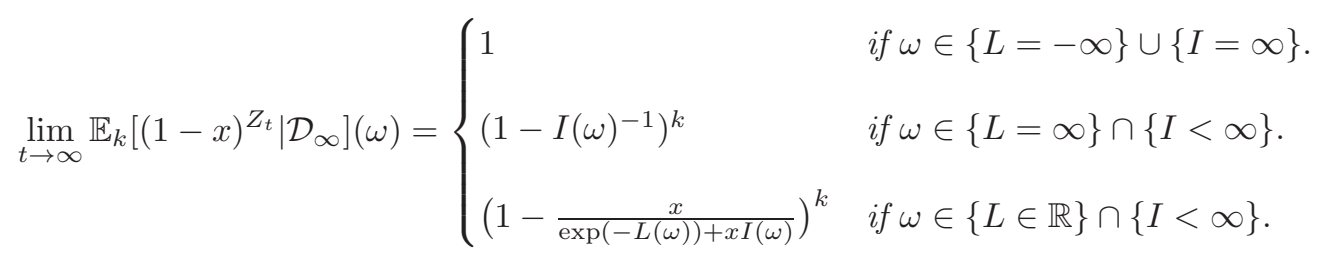

The third case occurs if and only if

$$
\int_{0}^{\infty}\left(b_{s}+d_{s}\right) d s<\infty \quad \text { and } \quad \prod_{k \geq 1} p\left(\tau_{k}(\omega)\right)>0
$$

Proof. By construction and monotonicity of $\left(I_{t}\right)$, these three cases cover all possible outcomes. The results follow by insertion into Lemma 4.9. In case 3. there is $m(\omega)<\infty$ such that $m(\omega) \geq e^{L_{t}(\omega)}$ for all $t$, since $\left(L_{t}(\omega)\right)_{t}$ converges in $\mathbb{R}$. Thus, almost surely

$$
\int_{0}^{\infty} b_{s} d s \leq \int_{0}^{\infty} m e^{-L_{s}} b_{s} d s=m I<\infty .
$$

Now, the convergence of $\left(L_{t}(\omega)\right)$ and the non-negativity of $d$ and $\log \frac{1}{p}$ give us that also $\int_{0}^{\infty} d_{s} d s$ as well as

$$
\int_{0}^{\infty} \log \frac{1}{p_{s}} d D_{s}(\omega)=-\sum_{k \geq 1} \log p_{\tau_{k}(\omega)}
$$

have to be finite, which shows that condition (4.11) is necessary for the third case. To see the sufficiency, from (4.11) the finiteness of $L(\omega)$ immediately follows analogously. Then, $\left(e^{-L_{t}(\omega)}\right)_{t}$ is bounded and thus by finiteness of $\int_{0}^{\infty} b_{s} d s$, also $I(\omega)$ has to be finite.

\section{Remark 4.12.}

1. The first part of condition (4.11) implies that with probability 1 there is only a finite number of birth and death events, while the second part offers either the possibility of $\lim _{t} D_{t}<\infty$ or $p$ converging to 1 on the support of $\kappa$, fast enough to compensate for $\left(D_{t}\right)$.

2. Only in the third case, the limiting probability generating function depends on $x$, which implies that, as soon as $b$ is bounded away from $0, \mathcal{Z}$ either goes extinct or explodes.

3. Since this Proposition provides results depending directly on the paths of $\left(D_{t}\right), b, d$ and $p$, it can easily be applied to random environments in the sense of choosing $b, d$ and/or $p$ to be stochastic processes.

4. Another possible generalization could be to drop the assertion that the limit $L$ exists. Then, we see that the first case still only holds if $\lim \sup _{t} L_{t}=-\infty$ or $I=\infty$. Secondly, in the case of $I<\infty$ we still obtain a limit independent of $x$, only if $\liminf _{t} L_{t}=\infty$. Hence, only the third case changes, where we obtain bounds on the limit in terms of $\liminf _{t} L_{t}$ and $\lim \sup _{t} L_{t}$.

Proof of Theorem ?.

First note that the assertions and Theorem 4.6 imply that almost surely

$$
L_{t}=\int_{0}^{t}\left(b_{s}-d_{s}\right) d s-\int_{0}^{t} \log \left(\frac{1}{p_{s}}\right) d D_{s} \sim \iota h(t) .
$$

(Since $h(t)=\Omega\left(t^{\alpha}\right)$ for some $\alpha>0$, in the case where $\beta \leq-1, \int_{0}^{t} \log \left(1 / p_{s}\right) d D_{s}$ has either a finite limit or it lies in $O\left(t^{\alpha / 2}\right) \subset o\left(t^{\alpha}\right)$ such that in either case it does not contribute to the asymptotics 
of $L_{t}$. Otherwise, it is asymptotically equivalent to $\int_{0}^{t} \log \left(1 / p_{s}\right) \kappa_{s} d s$.)

Now, we can apply Proposition 4.11

1.: If $\iota=1, L_{t} \stackrel{t \rightarrow \infty}{\longrightarrow} \infty$. Also, for almost every $\omega$ there is a $T(\omega) \in(0, \infty)$ such that $L_{t}(\omega) \geq$ $(1-\varepsilon) h(t)$ for all $t \geq T(\omega)$. Thus,

$$
I \leq I_{T}+\int_{T}^{\infty} e^{-(1-\varepsilon) h(s)} b_{s} d s<\infty .
$$

Hence, the second case of Proposition 4.11 concludes that

$$
\mathbb{P}\left(Z_{t} \stackrel{t \rightarrow \infty}{\longrightarrow} 0\right)=\lim _{t \rightarrow \infty} \mathbb{E}_{k}\left[(1-1)^{Z_{t}}\right]=\mathbb{E}\left[\left(1-I^{-1}\right)^{k}\right]<1 .
$$

2.: If $\iota=-1$, it is clear, that $L_{t} \stackrel{t \rightarrow \infty}{\longrightarrow}-\infty$ and independently of the integral condition of 2. the first part of Proposition 4.11 concludes

$$
\mathbb{P}\left(Z_{t} \stackrel{t \rightarrow \infty}{\longrightarrow} 0\right)=\mathbb{E}_{k}\left[\lim _{t \rightarrow \infty}(1-1)^{Z_{t}}\right]=1 .
$$

Otherwise, i.e. if $\iota=1$ but the integral condition holds, $L_{t} \stackrel{t \rightarrow \infty}{\longrightarrow} \infty$ and analogously to 1 . we find a finite random variable $T^{\prime}$ such that $L_{t} \leq(1+\varepsilon) h(t)$ for all $t \geq T^{\prime}$ almost surely. Thus,

$$
I=\int_{0}^{\infty} e^{-L_{s}} b_{s} d s \geq I_{T^{\prime}}+\int_{T^{\prime}}^{\infty} e^{-(1+\varepsilon) h(s)} b_{s} d s=\infty .
$$

Then again, the first part of Proposition 4.11 concludes the proof.

Remark 4.13 (Normalization function, rates of convergence). 1. There are two major cases in which a normalisation function $h$ as in Theorem 3 does not exist:

(a) The integral $\ell(t):=\int_{0}^{t}\left(b_{s}-d_{s}-\kappa_{s} \log \left(\frac{1}{p_{s}}\right)\right) d s$ converges to a constant. Then, $\mathcal{Z}$ will exhibit only a finite number of birth events almost surely and converge to a random variable, where the third part of Proposition 4.11 provides a way to compute the limiting distribution.

(b) The integral $\ell$ oscillates too strongly - e.g. $\ell(t)=t(1+\sin (t))$. This might happen in periodic models, which were briefly discussed in Kendall (1948). In this case, Lemma 4.9 still holds, while Proposition 4.11 as well as Theorem 4.6 do not apply.

2. In case 2. of Theorem 3 for the convergence rates of the survival probability conditioned on $\mathcal{D}_{\infty}$, the $\sigma$-algebra of the disaster times, we can estimate for arbitrary $k \geq 1$, using the processes $\left(X_{t}\right)$ and $\left(L_{t}\right)$ from Lemma 4.9 with $X_{0}=1$ and Bernoulli's inequality

$$
\begin{aligned}
-\frac{1}{h(t)} \log \mathbb{P}_{k}\left(Z_{t}>0 \mid \mathcal{D}_{\infty}\right) & =-\frac{1}{h(t)} \log \left(1-\left(1-X_{t}\right)^{k}\right) \\
& \sim-\frac{1}{h(t)} \log \left(X_{t}\right)=\frac{1}{h(t)} \log \left(e^{-L_{t}}+\int_{0}^{t} e^{-L_{s}} b_{s} d s\right) \\
& \sim \frac{1}{h(t)} \max \left\{-L_{t}, \log \left(\int_{0}^{t} e^{-L_{s}} b_{s} d s\right)\right\},
\end{aligned}
$$

where $\sim$ denotes asymptotic equivalence, i.e. $f \sim g \Leftrightarrow f(t) / g(t) \rightarrow 1$ as $t \rightarrow \infty$. Then, by Theorem 4.6, $\frac{L_{t}}{h(t)} \rightarrow \iota$, while for $0<\delta<\varepsilon$ and $t \geq t_{0}$ large enough it holds

$$
\int_{t_{0}}^{t} e^{-(1+\delta) h(s)} b_{s} d s \leq \int_{t_{0}}^{t} e^{-L_{s}} b_{s} d s \leq \int_{t_{0}}^{t} e^{-(1-\delta) h(s)} b_{s} d s .
$$

With more knowledge on $h$ and $b$, this approach can be used to compute bounds on the convergence rates. 


\section{A Large Deviations}

In Theorem [1, we make use of large deviations for Poisson processes. These can be e.g. read from Dembo and Zeitouni (1998), Exercise 5.2.12.

Lemma A.1 (Large deviations for a Poisson process). Let $P=\left(P_{t}\right)_{t \geq 0}$ be a unit rate Poisson process.

$$
\begin{aligned}
& \lim _{t \rightarrow \infty} \frac{1}{t} \log \mathbb{P}\left(P_{t} \geq x t\right)=-(1-x+x \log x) \text { for } x>1, \\
& \lim _{t \rightarrow \infty} \frac{1}{t} \log \mathbb{P}\left(P_{t} \leq x t\right)=-(1-x+x \log x) \text { for } x \in(0,1) .
\end{aligned}
$$

Moreover, for any $x \in(0,1)$

$$
\lim _{t \rightarrow \infty} \frac{1}{t} \log \mathbb{P}\left(P_{s} \leq x s \text { for all } s \leq t\right)=-(1-x+x \log x) .
$$

Proof. The first two assertions, A.1 and (A.2) are a consequence of Crámer's Theorem (Dembo and Zeitouni, 1998, Theorem 2.2.3, p.27). Moreover, the large deviation result (A.3) is an application of Dembo and Zeitouni (1998), Exercise 5.2.12, by rescaling and choosing $\phi(t)=x t$.

\section{References}

Athreya, K. B. and Kaplan, N. (1976). Limit theorems for a branching process with disasters. Journal of Applied Probability, 13(3):466-475.

Athreya, K. B. and Ney, P. E. (1972). Branching Processes. Springer.

Bansaye, V., Carlos, J., Millan, P., and Smadi, C. (2013). On the extinction of continuous state branching processes with catastrophes. Elec. J. Probab., 18(106):1-31.

Bartoszynski, R., Biihler, W. J., Chan, W., and Pearl, D. K. (1989). Population processes under the influence of disasters occurring independently of population size. J. Math. Biol., 27:167-178.

Bladt, M. and Nielsen, B. F. (2017). Regeneration and Harris Chains, pages 387-435. Springer US, Boston, MA.

Brockwell, P. J. (1985). The extinction time of a birth, death and catastrophe process and of a related diffusion model. Advances in Applied Probability, 17(1):42-52.

Brockwell, P. J., Gani, J., and Resnick, S. I. (1982). Birth, immigration and catastrophe processes. Advances in Applied Probability, 14(4):709-731.

Bühler, W. J. and Puri, P. S. (1989). The linear birth and death process under the influence of independently occurring disasters. Probability Theory and Related Fields, 83(1):59-66.

Casanova, A. G., Kurt, N., Wakolbinger, A., and Yuan, L. (2016). An individual-based model for the lenski experiment, and the deceleration of the relative fitness. Stochastic Processes and their Applications, 126(8):2211 - 2252.

Chaganty, N. R. and Sethuraman, J. (1993). Strong large deviation and local limit theorems. The Annals of Probability, 21(3):1671-1690.

Dembo, A. and Zeitouni, O. (1998). Large Deviations Techniques and Applications. Applications of mathematics. Springer.

Etheridge, A. (2001). An introduction to superprocesses. American Mathematical Society.

Ethier, S. N. and Kurtz, T. G. (1986). Markov processes. Characterization and convergence. Wiley Series in Probability and Mathematical Statistics: Probability and Mathematical Statistics. John Wiley \& Sons Inc., New York. 
Feller, W. (1971). An introduction to probability theory and its applications. Vol. II. Second edition. John Wiley \& Sons Inc., New York.

Harris, T. E. (1963). The theory of branching processes. Die Grundlehren der Mathematischen Wissenschaften, Bd. 119. Springer-Verlag, Berlin.

Jansen, S. and Kurt, N. (2014). On the notion(s) of duality for Markov processes. Probability Surveys, 11:59-120.

Kaplan, N., Sudbury, A., and Nilsen, T. S. (1975). A branching process with disasters. Journal of Applied Probability, 12(1):47-59.

Kendall, D. G. (1948). On the generalized 'birth-and-death' process. Ann. Math. Statist., 19(1):115.

Kumar, B. K., Vijayakumar, A., and Thilaka, B. (1998). Multitype branching processes with disasters ii: Total sojourn time and number of deaths. Mathematical and Computer Modelling, 28(11):103-114.

Lambert, A. (2008). Population dynamics and random genealogies. Stochastic Models, 24:45-163.

Pakes, A. G. (1986). The markov branching-castastrophe process. Stochastic Processes and their Applications, 23(1):1 - 33 .

Pakes, A. G. and Pollett, P. (1989). The supercritical birth, death and catastrophe process: limit theorems on the set of extinction. Stochastic Processes and their Applications, 32(1):161 - 170.

Peng, N., Pearl, D. K., Chan, W., and Bartoszyski, R. (1993). Linear birth and death processes under the influence of disasters with time-dependent killing probabilities. Stochastic Processes and their Applications, 45(2):243 - 258 .

Polfeldt, T. (1969). Integrating regularly varying functions with exponent -1. SIAM Journal on Applied Mathematics, 17(5):904-908.

Seneta, E. (1976). Regularly Varying Functions. Number Nr. 508 in Lecture Notes in Mathematics. Springer-Verlag.

Thilaka, B., Kumar, B., and Vijayakumar, A. (1998). Multitype branching processes with disasters I: The number of particles in the system. Mathematical and Computer Modelling, 28(11):87102. 